\title{
SEMILINEAR ELLIPTIC EQUATIONS IN THIN REGIONS WITH TERMS CONCENTRATING ON OSCILLATORY BOUNDARIES
}

\author{
JOSÉ M. ARRIETA*, ARIADNE NOGUEIRA ${ }^{\diamond}$ AND MARCONE C. PEREIRA ${ }^{\dagger}$
}

\begin{abstract}
In this work we study the behavior of a family of solutions of a semilinear elliptic equation, with homogeneous Neumann boundary condition, posed in a two-dimensional oscillating thin region with reaction terms concentrated in a neighborhood of the oscillatory boundary. Our main result is concerned with the upper and lower semicontinuity of the set of solutions. We show that the solutions of our perturbed equation can be approximated with one of a one-dimensional equation, which also captures the effects of all relevant physical processes that take place in the original problem.
\end{abstract}

\section{INTRODUCTION}

In this paper we investigate the behavior of a family of solutions given by a semilinear elliptic equation, with homogeneous Neumann boundary condition, defined in a two-dimensional oscillating thin region $R_{\varepsilon}$ with reaction terms concentrated in a neighborhood $o_{\varepsilon}$ of the oscillatory boundary of $R_{\varepsilon}$. We deal with an elliptic reaction-diffusion equation posed in the bounded open set $R_{\varepsilon}$ which degenerates into a line segment as the positive parameter $\varepsilon$ goes to zero. Also, we assume that the reaction of the model only occur in a narrow strip $o_{\varepsilon}$ close to the border, which also can present high oscillatory structure. See Figure 1 below which illustrates the open region $R_{\varepsilon}$ and the narrow neighborhood $o_{\varepsilon}$ mentioned here.

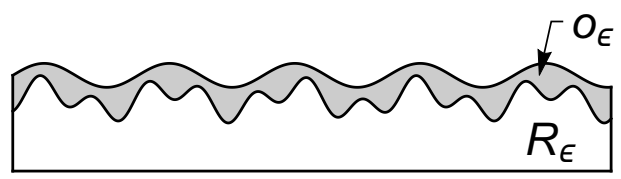

FiguRE 1 . The thin domain $R_{\varepsilon}$ and the strip $o_{\varepsilon}$ where reactions take place.

Our main result is that the family of solutions are upper and lower semicontinuous at $\varepsilon=0$. Indeed, we show that the starting singular equation defined in the two-dimensional region can be approximated with one which is a one-dimensional regular equation, which captures the effects of all relevant physical processes that take place in the original problem. Therefore, the limit equation will preserve features of the original system, giving conditions to access the qualitative behavior of the modeled problem in a simpler way.

Let us recall that elliptic boundary value problem models diffusion and interactions among agents which can be cells, amount of chemicals or biological organisms. Thus, we are supposing here that the agents are located in an extremely thin region with reactions taking place just in a small neighborhood of the border. It is worth noting that our model includes the possibility that the thin region as well the narrow neighborhood present high oscillatory behavior, modeling complex regions of interactions.

Indeed, we are dealing with phenomenas which are posed in very thin channels and that also may present several heterogeneities. In a precise way, we establish the effective behavior of the process by neglecting the squeezing of the region as well as the fluctuations due to the heterogeneities on the boundary. At the end, we get the homogenized model whose behavior must be as closer as possible to the original one.

2010 Mathematics Subject Classification. 34B15, 35J75, 35J91.

Key words and phrases. Semilinear elliptic equations, singular elliptic equations, upper semicontinuity, lower semicontinuity, thin domains, concentrating terms. 
Potential applications of our modeling are in fields such as lubrication, nanotechnology, fluid-structure interaction mechanism in vascular dynamics, management and control of aquatic ecological systems and so on. See for instance [1, 2, 3, 4, 5, 6, 7, 8, where theoretical and practical aspects of mathematical modeling and applications in this context are investigated. In particular, it is well known from the literature of ecological modeling that lakes with complex geometry show larger spatial variability of limnological parameters than lakes with a simple shape 9 . Thus, it is important analyzing models where a larger class of roughness and thickness of the domain are allowed.

From the numerical point of view, it is not difficult to see that the combination of the squeezing channel and heterogeneities on the boundary makes hard a direct numerical simulation of the model. In fact, a very fine discretization mesh would be needed meaning a significant time of computation. On the other side, the homogenized model obtained is a one-dimensional regular equation where no numerical difficult appears. Moreover, it is known, for instance from [10, 11] and references therein, that homogenized equations are good approximations to heterogeneous problems in the sense that they describe global and essential properties of the modeled phenomena.

There are several works dealing with partial differential equations posed in thin domains. We first mention the pioneering works [12, 13, as well the subsequent papers [14, 15, 16], where the authors investigate the asymptotic behavior of dynamical systems given by a class of semilinear parabolic equations in thin domains of $\mathbb{R}^{n}, n \geq 2$. We also cite [17, 18, where the $p$-Laplacian problem in thin regions is considered, and 19, which studies a linear elliptic problem in perforated thin domains with rapidly varying thickness. In [20] the authors consider nonlinear monotone problems in a multidomain with a highly oscillating boundary. In $21,22,23,24,25,26,27,28$ and references therein, we have recently studied many classes of oscillating thin regions for elliptic and parabolic equations with Neumann boundary conditions, discussing limit problems and convergence properties. For nonlocal equations in thin structures we also mention [29, 30, 31, 32.

On the other hand, there are many works in the literature concerned with singular elliptic and parabolic problems featuring potential and reactions terms concentrated in a small neighborhood of a portion of the boundary. In fixed bounded domains, we cite the pioneering works [33, 34, 35. In regions presenting oscillatory behavior, we mention the recent ones [36, 37. In [38, 39] we also have studied problems allowing narrow strips with oscillatory border in fixed bounded open sets.

Our main goal here is to discuss a model combining these both singular situations (the thin domain problem and concentrated reactions) in a more general framework. For this, we generalize [40] adapting methods and techniques developed in [33] to deal with a larger class of concentrated integrals and thin sets getting appropriate estimates which must be uniform for any $\varepsilon$ close to zero. As we will see, this is not a trivial task. Indeed, a non-standard functional spaces as Lebesgue-Bochner spaces have to be considered in a context of nonlinear analysis, compact convergence and Fixed Point Theorem in such a way that we are able to estimate our solutions. Finally, we pass to the limit in the model obtaining its asymptotic behavior at $\varepsilon=0$. As we have already mentioned, the limit equation will explicitly show the effect of both the oscillatory behavior and geometry of the regions where the differential equation is defined.

The paper is organized as follows: in Section 2, we set our assumptions, notations and state the main result concerning to the upper and lower semicontinuity of the set of solutions. In Section 3, we introduce our functional setting, and obtain results which allow us to estimate the concentrated integrals. In Section 4. we deal with nonlinear maps related with the nonlinear reaction terms of the equation, and in Section 5 . we show our main result getting the asymptotic behavior of the solutions at $\varepsilon=0$.

\section{Assumptions, NOtATIONS AND MAIN RESUlt}

Let us consider the following semilinear elliptic equation with homogeneous Neumann boundary conditions

$$
\begin{cases}-\Delta v^{\varepsilon}+v^{\varepsilon}=\frac{1}{\varepsilon} \chi^{o_{\varepsilon}} f\left(v^{\varepsilon}\right) & \text { in } R_{\varepsilon} \\ \frac{\partial v^{\varepsilon}}{\partial \nu^{\varepsilon}}=0 & \text { on } \partial R_{\varepsilon}\end{cases}
$$


where for each $\varepsilon>0, R_{\varepsilon} \subset \mathbb{R}^{2}$ is an oscillating thin domain given by

$$
R_{\varepsilon}=\{(x, y) ; 0<x<1,0<y<\varepsilon g(x / \varepsilon)\} .
$$

The vector $\nu^{\varepsilon}=\left(\nu_{1}^{\varepsilon}, \nu_{2}^{\varepsilon}\right)$ denotes the unit outward normal vector to the boundary $\partial R_{\varepsilon}, \partial / \partial \nu^{\varepsilon}$ is the normal derivative, and $\chi^{o_{\varepsilon}}$ is the characteristic function of the set $o_{\varepsilon}$ defined by

$$
o_{\varepsilon}=\left\{(x, y) ; 0<x<1, \varepsilon\left(g(x / \varepsilon)-\varepsilon h\left(x / \varepsilon^{\beta}\right)\right)<y<\varepsilon g(x / \varepsilon)\right\} .
$$

We assume

(i) $\beta>0$;

(ii) the nonlinearity $f: \mathbb{R} \rightarrow \mathbb{R}$ is a $\mathcal{C}^{2}$ function;

(iii) the functions $g, h:(0,1) \rightarrow \mathbb{R}$ are positive, $L_{g}$ and $L_{h}$-periodic, respectively, and possess $g_{0}, g_{1}, h_{0}, h_{1} \in$ $\mathbb{R}$ such that

$$
0<g_{0} \leq g(x) \leq g_{1}<\infty, \quad 0 \leq h_{0} \leq h(x) \leq h_{1}<\infty, \quad \forall x \in(0,1) ;
$$

(iv) $g$ has bounded derivative.

Remark 2.1. Notice that, calling $g_{\varepsilon}(x)=g(x / \varepsilon)$ and $h_{\varepsilon}(x)=h\left(x / \varepsilon^{\beta}\right)$, it follows from [11, Theorem 2.6] that there exist $\mu_{g}, \mu_{h} \in \mathbb{R}$ such that

$$
g_{\varepsilon} \stackrel{*}{\rightarrow} \mu_{g}=\frac{1}{L_{g}} \int_{0}^{L_{g}} g(s) d s \quad \text { and } \quad h_{\varepsilon} \stackrel{*}{\rightarrow} \mu_{h}=\frac{1}{L_{h}} \int_{0}^{L_{h}} h(s) d s \quad \text { in } L^{\infty}(0,1) .
$$

The constants $\mu_{g}$ and $\mu_{h}$ are the average of the periodic functions $g$ and $h$ respectively.

Let us emphasize that $R_{\varepsilon} \subset(0,1) \times\left(0, \varepsilon g_{1}\right)$ is a two-dimensional thin region with oscillatory boundary which degenerates to the unit interval as $\varepsilon \rightarrow 0$. Also, $o_{\varepsilon} \subset R_{\varepsilon}$ represents an oscillating $\varepsilon$-neighborhood to the upper boundary of $R_{\varepsilon}$ where the reaction term takes place.

Notice that here, we are in agreement with [33. We combine the characteristic function $\chi^{o_{\varepsilon}}$ and the positive parameter $\varepsilon$ in order to set concentration of reactions on the small strip $o_{\varepsilon} \subset R_{\varepsilon}$ through the term

$$
\frac{1}{\varepsilon} \chi^{o_{\varepsilon}} \in L^{\infty}\left(R_{\varepsilon}\right)
$$

We will show that, in a certain functional setting, the family of solutions from the perturbed problem (2.1) converges to a solution of a one-dimensional equation of the same type, with homogeneous Neumann boundary condition, capturing the variable profile of the domain $R_{\varepsilon}$ as well as the oscillatory behavior of the neighborhood $o_{\varepsilon}$. Indeed, we obtain the following limit problem

$$
\left\{\begin{array}{l}
-q_{0} u_{x x}+u=f_{0}(u) \quad \text { in }(0,1) \\
u_{x}(0)=u_{x}(1)=0
\end{array}\right.
$$

with

$$
q_{0}=\frac{1}{\left|Y^{*}\right|} \int_{Y^{*}}\left\{1-\frac{\partial X}{\partial y_{1}}\left(y_{1}, y_{2}\right)\right\} d y_{1} d y_{2} \quad \text { and } \quad f_{0}(\cdot)=\frac{L_{g}}{\left|Y^{*}\right|} \mu_{h} f(\cdot) .
$$

The function $X$ is the unique solution of the auxiliary problem

where $Y^{*}$ given by

$$
\left\{\begin{array}{l}
-\Delta X=0 \text { in } Y^{*} \\
\frac{\partial X}{\partial N}=0 \text { in } B_{2} \\
\frac{\partial X}{\partial N}=N_{1} \text { in } B_{1} \\
X \text { is } L_{g} \text {-periodic in } y_{1} \\
\int_{Y^{*}} X d y_{1} d y_{2}=0
\end{array}\right.
$$

$$
Y^{*}=\left\{\left(y_{1}, y_{2}\right) \in \mathbb{R}^{2} ; 0<y_{1}<L_{g}, 0<y_{2}<g\left(y_{1}\right)\right\}
$$

is the representative cell of the thin region $R_{\varepsilon}$. The vector $N=\left(N_{1}, N_{2}\right)$ is the outward normal vector to the boundary $\partial Y^{*}$ with $B_{1}$ and $B_{2}$ denoting the upper and lower boundary of $\partial Y^{*}$ respectively. 
We first notice that the diffusion coefficient $q_{0}$, usually called homogenized coefficient, is a positive constant by [21, Theorem 4.3]. Besides, it exhibits the effect of the geometry and the oscillatory behavior of the thin region since depends on the auxiliary solution $X$ which is set in the representative cell $Y^{*}$. On the other side, nonlinearity $f_{0}$ captures the influence of the concentration neighborhood on the reaction $f$ by the average $\mu_{h}$, and also the effect of the geometry and oscillatory behavior of $R_{\varepsilon}$ by the term $L_{g} /\left|Y^{*}\right|$. The limit problem 2.2 is often called homogenized equation. Consequently, from homogenization and asymptotic analysis results [10, 11, 41, 42, 43, we can say that 2.2 establishes the global and essential properties of the original model 2.1 being a good and non-singular approximation.

It is worth noting that the results obtained here generalize the ones from [40] since the thin domain analyzed there does not exhibit any oscillatory behavior. Furthermore, we emphasize that our task is not easy here. In order to accomplish our goal, we have to be able to estimate the solutions in very small neighborhoods of the oscillatory boundary in such way that we can pass to the limit at $\varepsilon=0$.

The solutions of our problem are defined in open sets which varies with respect to parameter $\varepsilon>0$. Thus, the first step in our analysis is to set an approach in order to face this domain perturbation problem. Here we adopt the same strategy used, for instance, in [21]. We rescale the thin region $R_{\varepsilon}$ keeping the $x$-coordinate and multiplying the values of $y$ by a factor $1 / \varepsilon$ avoiding the thin domain situation. Performing this change of variable, we obtain the following problem:

$$
\left\{\begin{array}{c}
-\frac{\partial^{2} u^{\varepsilon}}{\partial x_{1}^{2}}-\frac{1}{\varepsilon^{2}} \frac{\partial^{2} u^{\varepsilon}}{\partial x_{2}^{2}}+u^{\varepsilon}=\frac{1}{\varepsilon} \chi^{\theta_{\varepsilon}} f\left(u^{\varepsilon}\right) \quad \text { in } \Omega_{\varepsilon} \\
\frac{\partial u^{\varepsilon}}{\partial x_{1}} N_{1}^{\varepsilon}+\frac{1}{\varepsilon^{2}} \frac{\partial u^{\varepsilon}}{\partial x_{2}} N_{2}^{\varepsilon}=0 \quad \text { on } \partial \Omega_{\varepsilon}
\end{array}\right.
$$

where $N^{\varepsilon}=\left(N_{1}^{\varepsilon}, N_{2}^{\varepsilon}\right)$ is the outward normal vector to the boundary $\partial \Omega_{\varepsilon}$,

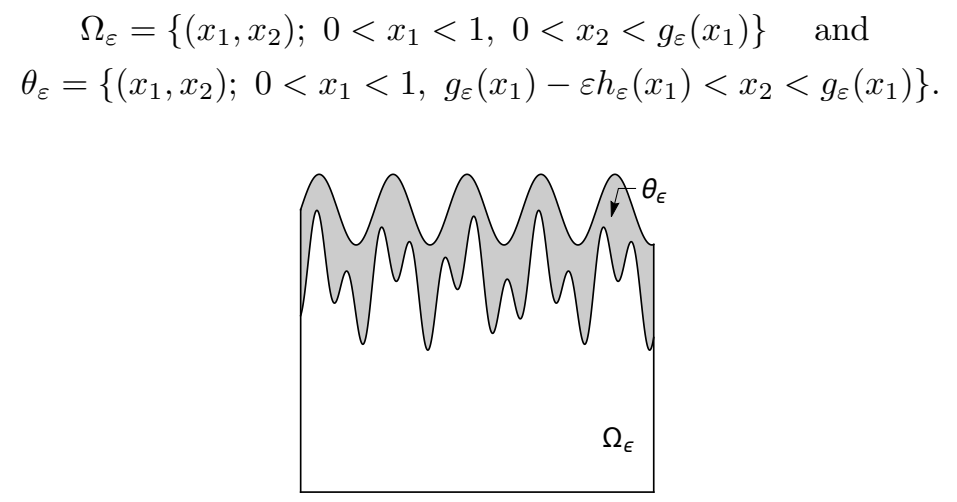

FiguRE 2. The modified domain $\Omega_{\varepsilon}$ and the neighborhood $\theta_{\varepsilon}$.

It is not difficult to see that problems 2.1 and 2.4 are equivalent. In some sense, we have rescaled the neighborhood $o_{\varepsilon}$ into the strip $\theta_{\varepsilon} \subset \Omega_{\varepsilon}$ and substituted the thin region $R_{\epsilon}$ by the oscillating domain $\Omega_{\varepsilon}$, at a cost of introducing a very strong diffusion mechanism in the $x_{2}$-direction given by the factor $1 / \varepsilon^{2}$. This will make the solutions from 2.4 to become more and more homogeneous in this direction as $\varepsilon$ goes to zero. In this way, the limit solution will not depend on $x_{2}$, and therefore, the limit equation will be one dimensional. Notice that is in full agreement with the intuitive idea that a partial differential equation posed in a thin domain should approach one defined in a line segment.

In order to obtain our convergence results, we have to compare functions defined in different functional spaces. Fixed $1 / 2<s<1$, we consider the Lebesgue-Bochner spaces

$$
X=L^{2}\left(0,1 ; H^{s}\left(0, g_{1}\right)\right) \quad \text { and } \quad X_{\varepsilon}=L^{2}\left(0,1 ; H^{s}\left(0, g_{\varepsilon}\left(x_{1}\right)\right)\right)
$$

which will be discussed in Section 3, as well as the Hilbert space $X_{0}=L^{2}(0,1)$ with the norm given by

$$
\|u\|_{X_{0}}=\sqrt{\mu_{g}}\|u\|_{L^{2}(0,1)}
$$


The perturbed problem (2.1) will be set in $X_{\varepsilon}$, and the limit equation 2.2 in $X_{0}$.

Since $X_{0} \subset X_{\varepsilon}$, we can consider the operator

$$
\begin{aligned}
E_{\varepsilon}: & X_{0} \rightarrow X_{\varepsilon} \\
u\left(x_{1}\right) & \mapsto\left(E_{\varepsilon} u\right)\left(x_{1}, x_{2}\right)=u\left(x_{1}\right)
\end{aligned}
$$

which satisfies

$$
\begin{aligned}
\left\|E_{\varepsilon} u\right\|_{X_{\varepsilon}}^{2} & =\int_{0}^{1}\left\|\left(E_{\varepsilon} u\right)\left(x_{1}, \cdot\right)\right\|_{H^{s}\left(0, g_{\varepsilon}\left(x_{1}\right)\right)}^{2} d x_{1}=\int_{0}^{1} g_{\varepsilon}\left(x_{1}\right)\left|u\left(x_{1}\right)\right|^{2} d x_{1} \\
& \rightarrow \mu_{g}\|u\|_{L^{2}(0,1)}=\|u\|_{X_{0}}^{2}, \quad \text { as } \varepsilon \rightarrow 0 .
\end{aligned}
$$

As in [43, we obtain an appropriate way to compare solutions from 2.4) and 2.2.

Definition 2.2. We say that $\left\{u^{\varepsilon}\right\}_{\varepsilon>0} \subset X_{\varepsilon}$ E-converges to a function $u \in X_{0}$, if $\left\|u^{\varepsilon}-E_{\varepsilon} u\right\|_{X_{\varepsilon}} \rightarrow 0$ as $\varepsilon \rightarrow 0$, where $E_{\varepsilon}$ is given by 2.5 . It is denoted by $u_{\varepsilon} \stackrel{E}{\rightarrow} u$.

This notion of convergence can be also extended to sets in the following manner: let $J_{\varepsilon}$ be a family of sets in $X_{\varepsilon}$. We say that $J_{\varepsilon} \subset X_{\varepsilon}$ is

(i) upper semicontinuous at $\varepsilon=0$, if $\operatorname{dist}_{H}\left(J_{\varepsilon}, E_{\varepsilon} J_{0}\right) \stackrel{\varepsilon \rightarrow 0}{\longrightarrow} 0$;

(ii) lower semicontinuous at $\varepsilon=0$, if $\operatorname{dist}_{H}\left(E_{\varepsilon} J_{0}, J_{\varepsilon}\right) \stackrel{\varepsilon \rightarrow 0}{\longrightarrow} 0$.

Here, $\operatorname{dist}_{H}(A, B)$ denotes the Hausdorff semi-distance given by

$$
\operatorname{dist}_{H}(A, B)=\sup _{x \in A} \inf _{y \in B}\|x-y\|_{X_{\varepsilon}} .
$$

Remark 2.3. Also, the following characterizations are very useful:

(i) The family $\left\{J_{\varepsilon}\right\}$ is upper semicontinuous at $\varepsilon=0$ if every sequence $\left\{u_{\varepsilon}\right\}$, with $u_{\varepsilon} \in J_{\varepsilon}$ and $\varepsilon \rightarrow 0$, has a subsequence E-convergent to an element of $J_{0}$;

(ii) The family $\left\{J_{\varepsilon}\right\}$ is lower semicontinuous at $\varepsilon=0$ if $J_{0}$ is compact and for all $u \in J_{0}$ exists a sequence $\left\{u_{\varepsilon}\right\}$, with $u_{\varepsilon} \in J_{\varepsilon}$ and $\varepsilon \rightarrow 0$, such that $u_{\varepsilon} \stackrel{E}{\rightarrow} u$.

Finally, let us consider, for $0<\varepsilon \leq \varepsilon_{0}$, the folowing sets given by problem 2.4

$$
\mathcal{E}_{\varepsilon, R}=\left\{u^{\varepsilon} \in H^{1}\left(\Omega_{\varepsilon}\right) ; u^{\varepsilon} \text { is a solution of } 2.4 \text { and }\left\|u^{\varepsilon}\right\|_{L^{\infty}\left(\Omega_{\varepsilon}\right)} \leq R\right\}
$$

and

$$
\mathcal{E}_{0, R}=\left\{u \in H^{1}(0,1) ; u \text { is a solution of } 2.2 \text { and }\|u\|_{L^{\infty}(0,1)} \leq R\right\} .
$$

Now, we state our main result which concerns on upper and lower semicontinuity of the set $\mathcal{E}_{\varepsilon, R}$ at $\varepsilon=0$.

Theorem 2.4. (a) For any sequence $u_{\varepsilon} \in \mathcal{E}_{\varepsilon, R}$, with $\varepsilon \rightarrow 0$, there is a subsequence (also denoted by $u_{\varepsilon}$ ) and $u_{0} \in \mathcal{E}_{0, R}$ such that $u_{\varepsilon} \stackrel{E}{\rightarrow} u_{0}$ in $X_{\varepsilon}$ when $\varepsilon \rightarrow 0$.

(b) For any hyperbolic equilibrium point $u_{0} \in \mathcal{E}_{0, R}$, there is sequence $u_{\varepsilon} \in \mathcal{E}_{\varepsilon, R}$ such that $u_{\varepsilon} \stackrel{E}{\rightarrow} u_{0}$ in $X_{\varepsilon}$ as $\varepsilon \rightarrow 0$.

Remark 2.5. Recall that a solution $u$ of a boundary value problem is hyperbolic if $\lambda=0$ is not an eigenvalue of the linearized problem around $u$. In other words, $u \in \mathcal{E}_{0, R}$ is hyperbolic if $\lambda=0$ is not an eigenvalue of the eigenvalue problem

$$
\left\{\begin{array}{c}
-q_{0} v_{x x}+v=\partial_{u} f_{0}(u) v+\lambda v \quad \text { in }(0,1) \\
v_{x}(0)=v_{x}(1)=0
\end{array}\right.
$$

where $q_{0}$ and $f_{0}$ are defined in 2.3.

Remark 2.6. Since we are concerned with solutions which are uniformly bounded in $L^{\infty}(\Omega)$, we may take $f$ of class $\mathcal{C}^{2}$ bounded with bounded derivatives. In fact, we may perform a cut-off in $f$ outside the region $|u| \leq R$ without modifying any of these solutions (see for instance [42, Remark 2.2] or [45, Remark 2.2]).

Remark 2.7. The assertions $(a)$ and $(b)$ in Theorem 2.4 respectively mean upper and lower semicontinuity of the equilibria set to the parabolic problem associated with (2.4) at $\epsilon=0$. 


\section{Functional SPACES AND CONCEntrated InTEGRAls}

In this section, we first establish the functional spaces used to analyze the concentrated integrals. Next, we perform some estimates in such functional spaces.

Definition 3.1. Let $s=m+\sigma>0$, with $m=0,1, \ldots, 0 \leq \sigma<1$ and let $O \subset R^{n}$ a domain. For $1 \leq p<\infty$, we call fractional Sobolev space $W^{s, p}(O)$ the space of functions $u$ such that

(i) $u \in W^{m, p}(O)$ if $\sigma=0$,

(ii) $u \in W^{m, p}(O)$ and

$$
\iint_{O \times O} \frac{\left|\partial^{\alpha} u(x)-\partial^{\alpha} u(y)\right|^{p}}{|x-y|^{n+\sigma p}} d x d y<\infty
$$

if $\sigma>0$.

The norm in $W^{s, p}(O)$, that makes it Banach, is:

$$
\|u\|_{W^{m, p}(O)}^{p}=\sum_{|\alpha| \leq m} \int_{O}\left|\partial^{\alpha} u\right|^{p} d x \text { in the case }(i)
$$

and

$$
\|u\|_{W^{s, p}(O)}^{p}=\|u\|_{W^{m, p}(O)}^{p}+\sum_{|\alpha|=m} \iint_{O \times O} \frac{\left|\partial^{\alpha} u(x)-\partial^{\alpha} u(y)\right|^{p}}{|x-y|^{n+\sigma p}} d x d y \text { in the case (ii). }
$$

Furthermore, if $p=2$ we call it $H^{s}(O)$ and it is a Hilbert space.

Now let us follow [50] to introduce what we call Lebesgue and Sobolev-Bochner generalized spaces. They are a natural generalization to Lebesgue and Sobolev spaces using Bochner integrals. The usual Lebesgue and Sobolev-Bochner spaces may be found for instance in [41, 52].

Let us consider a function $G:(0,1) \rightarrow \mathbb{R}$ satisfying that there exist $0<G_{0} \leq G_{1}$ with $G_{0} \leq G(x) \leq G_{1}$.

Definition 3.2. Let us consider a function $G:(0,1) \rightarrow \mathbb{R}$ satisfying $0<G_{0} \leq G(x) \leq G_{1}$ for some constants $0<G_{0} \leq G_{1}$. Let $1 \leq p \leq \infty, 1 \leq q<\infty$. The Lebesgue-Bochner generalized spaces, denoted by $L^{p}\left(0,1 ; L^{q}\left(0, G\left(x_{1}\right)\right)\right)$, are defined by

$$
L^{p}\left(0,1 ; L^{q}\left(0, G\left(x_{1}\right)\right)\right):=\left\{u: \Omega_{\varepsilon} \rightarrow \mathbb{R} \text { measurable } ; u\left(x_{1}, \cdot\right) \in L^{q}\left(0, G\left(x_{1}\right)\right) \text { for almost every } x \in \Omega\right\}
$$

and they are Banach spaces with the norm

$$
\|u\|_{L^{p}\left(0,1 ; L^{q}\left(0, G\left(x_{1}\right)\right)\right)}= \begin{cases}\left(\int_{0}^{1}\left\|u\left(x_{1}, \cdot\right)\right\|_{L^{q}\left(0, G\left(x_{1}\right)\right)}^{p} d x_{1}\right)^{1 / p}, & p<\infty \\ \operatorname{ess} \sup _{x \in(0,1)}\left\|u\left(x_{1}, \cdot\right)\right\|_{L^{q}\left(0, G\left(x_{1}\right)\right)}, & p=\infty .\end{cases}
$$

When $p=q=2$ such space is Hilbert with the inner product

$$
(u, v)_{L^{2}\left(0,1 ; L^{2}\left(0, G\left(x_{1}\right)\right)\right.}=\int_{0}^{1}\left(u\left(x_{1}, \cdot\right), v\left(x_{1}, \cdot\right)\right)_{L^{2}\left(0, G\left(x_{1}\right)\right)} d x_{1} .
$$

Remark 3.3. Since $q<\infty$, the function $x_{1} \mapsto\left\|u\left(x_{1}, \cdot\right)\right\|_{L^{q}\left(0, G\left(x_{1}\right)\right)}$ is measurable by Fubini's Theorem. Then the space $L^{p}\left(0,1 ; L^{q}\left(0, G\left(x_{1}\right)\right)\right.$ is well defined.

Analogously, the Sobolev-Bochner generalized spaces, denoted by $L^{p}\left(0,1 ; W^{s, q}\left(0, G\left(x_{1}\right)\right)\right)$ for $s>0$, are defined by

$$
L^{p}\left(0,1 ; W^{s, q}\left(0, G\left(x_{1}\right)\right)\right):=\left\{u \in L^{p}\left(0,1 ; L^{q}\left(0, G\left(x_{1}\right)\right)\right) ; u\left(x_{1}, \cdot\right) \in W^{s, q}\left(0, G\left(x_{1}\right)\right)\right\} .
$$


Such spaces are Banach with the norm

$$
\|u\|_{L^{p}\left(0,1 ; W^{s, q}\left(0, G\left(x_{1}\right)\right)\right)}= \begin{cases}\left(\int_{0}^{1}\left\|u\left(x_{1}, \cdot\right)\right\|_{W^{s, q}\left(0, G\left(x_{1}\right)\right)}^{p} d x_{1}\right)^{1 / p}, & p<\infty, \\ \operatorname{ess} \sup _{x_{1} \in(0,1)}\left\|u\left(x_{1}, \cdot\right)\right\|_{W^{s, q}\left(0, G\left(x_{1}\right)\right)}, & p=\infty,\end{cases}
$$

and, again, they are Hilbert spaces if $p=q=2$.

In general, it follows from [41, Proposition 3.59] that, if $H$ is a Hilbert space and $1 \leq p<\infty$, then the dual space of $L^{p}(0,1 ; H)$ is given by

$$
\left[L^{p}(0,1 ; H)\right]^{\prime}=L^{q}\left(0,1 ; H^{\prime}\right),
$$

where $H^{\prime}$ is the dual space of $H$ and $p, q$ are conjugates.

In our case we will consider the family of Lebesgue and Sobolev-Bochner generalized space for the function $G\left(x_{1}\right)=g_{\varepsilon}\left(x_{1}\right)$, with $g_{\varepsilon}$ as in Section 2 (see Remark 2.1).

3.1. Some technical results. Next we will get some non-trivial properties which are important in our context. First, we construct a unidimensional extension operator that will help us to work with different definitions of Sobolev fractional spaces, making their norms equivalent.

Lemma 3.4. Fix $\varepsilon>0$ and $x_{1} \in(0,1)$, if we call $I_{\varepsilon}=\left(0, g_{\varepsilon}\left(x_{1}\right)\right)$, with $g_{\varepsilon}$ as in Section 2 (see Remark 2.1), then there exists a continuous linear extension operator $P: L^{2}\left(I_{\varepsilon}\right) \rightarrow L^{2}(\mathbb{R})$ such that $P u=u$ in $I_{\varepsilon}$, with $\|P u\|_{L^{2}(\mathbb{R})} \leq \lambda_{0}\|u\|_{L^{2}\left(I_{\varepsilon}\right)},\|P u\|_{H^{s}(\mathbb{R})} \leq \lambda_{s}\|u\|_{H^{s}\left(I_{\varepsilon}\right)}$ and $\|P u\|_{H^{1}(\mathbb{R})} \leq \lambda_{1}\|u\|_{H^{1}\left(I_{\varepsilon}\right)}$, for $0<s<1$, where the constants $\lambda_{0}, \lambda_{s}, \lambda_{1} \geq 1$ are independent of $\varepsilon>0$ and $x_{1} \in(0,1)$.

Proof. Notice that $I_{0}:=\left(0, g_{0}\right) \subset I_{\varepsilon}$, for all $\varepsilon>0$. The construction of the extension operator will be in two steps: first we will extend the functions from $I_{\varepsilon}$ into $I=\left(0, g_{1}\right)$. Next, from interval $I$ into $\mathbb{R}$.

If $2 g_{0} \geq g_{1}$, we define $P_{\varepsilon}$ by a reflection procedure. If $\varphi \in L^{2}\left(I_{\varepsilon}\right)$,

$$
\left(P_{\varepsilon} \varphi\right)(y)=\left\{\begin{array}{ll}
\varphi(y), & \text { if } y \in I_{\varepsilon} \\
\varphi\left(2 g_{\varepsilon}\left(x_{1}\right)-y\right), & \text { if } y \in I \backslash I_{\varepsilon}
\end{array} .\right.
$$

Let us see that $P_{\varepsilon}$ is well defined, that is, that $\left(2 g_{\varepsilon}\left(x_{1}\right)-y\right) \in I_{\varepsilon}$ if $y \in I \backslash I_{\varepsilon}$. Indeed if $y \in I \backslash I_{\varepsilon}$,

$$
\left\{\begin{array}{l}
2 g_{\varepsilon}\left(x_{1}\right)-y>2 g_{\varepsilon}\left(x_{1}\right)-g_{1} \geq 2 g_{\varepsilon}\left(x_{1}\right)-2 g_{0}>0 \\
2 g_{\varepsilon}\left(x_{1}\right)-y<2 g_{\varepsilon}\left(x_{1}\right)-g_{\varepsilon}\left(x_{1}\right)=g_{\varepsilon}\left(x_{1}\right)
\end{array}\right.
$$

then $\left(2 g_{\varepsilon}\left(x_{1}\right)-y\right) \in I_{\varepsilon}$.

Now, let us show the continuity of the operator. If $\varphi \in H^{1}\left(I_{\varepsilon}\right)$,

$$
\left\|P_{\varepsilon} \varphi\right\|_{L^{2}(I)}^{2}=\|\varphi\|_{L^{2}\left(I_{\varepsilon}\right)}^{2}+\int_{2 g_{\varepsilon}\left(x_{1}\right)-g_{1}}^{g_{\varepsilon}\left(x_{1}\right)}|\varphi(z)|^{2} d z \leq\|\varphi\|_{L^{2}\left(I_{\varepsilon}\right)}^{2}+\int_{2 g_{0}-g_{1}}^{g_{\varepsilon}\left(x_{1}\right)}|\varphi(z)|^{2} d z \leq 2\|\varphi\|_{L^{2}\left(I_{\varepsilon}\right)}^{2} .
$$

Besides

$$
\begin{aligned}
\left\|\frac{\partial P_{\varepsilon} \varphi}{\partial y}\right\|_{L^{2}(I)}^{2} & =\int_{I_{\varepsilon}}\left|\frac{\partial \varphi}{\partial y}\right|^{2}+\int_{g_{\varepsilon}\left(x_{1}\right)}^{g_{1}}\left|\frac{\partial}{\partial y} \varphi\left(2 g_{\varepsilon}\left(x_{1}\right)-y\right)\right|^{2} d y \\
& \leq\left\|\frac{\partial \varphi}{\partial y}\right\|_{L^{2}\left(I_{\varepsilon}\right)}^{2}+\int_{2 g_{0}-g_{1}}^{g_{\varepsilon}\left(x_{1}\right)}\left|\frac{\partial \varphi}{\partial y}(z)\right|^{2} d z \leq 2\left\|\frac{\partial \varphi}{\partial y}\right\|_{L^{2}\left(I_{\varepsilon}\right)}^{2} .
\end{aligned}
$$

For $0<s<1$, we have

$$
\left\|P_{\varepsilon} \varphi\right\|_{H^{s}(I)}^{2}=\left\|P_{\varepsilon} \varphi\right\|_{L^{2}(I)}^{2}+\iint_{I \times I} \frac{\left|P_{\varepsilon} \varphi\left(y_{1}\right)-P_{\varepsilon} \varphi\left(y_{2}\right)\right|^{2}}{\left|y_{1}-y_{2}\right|^{1+2 s}} d y
$$




$$
\begin{aligned}
& \leq 2\|\varphi\|_{L^{2}\left(I_{\varepsilon}\right)}^{2}+\iint_{I_{\varepsilon} \times I_{\varepsilon}} \frac{\left|P_{\varepsilon} \varphi\left(y_{1}\right)-P_{\varepsilon} \varphi\left(y_{2}\right)\right|^{2}}{\left|y_{1}-y_{2}\right|^{1+2 s}} d y+\iint_{A} \frac{\left|P_{\varepsilon} \varphi\left(y_{1}\right)-P_{\varepsilon} \varphi\left(y_{2}\right)\right|^{2}}{\left|y_{1}-y_{2}\right|^{1+2 s}} d y \\
& \quad+\iint_{B} \frac{\left|P_{\varepsilon} \varphi\left(y_{1}\right)-P_{\varepsilon} \varphi\left(y_{2}\right)\right|^{2}}{\left|y_{1}-y_{2}\right|^{1+2 s}} d y+\iint_{C} \frac{\left|P_{\varepsilon} \varphi\left(y_{1}\right)-P_{\varepsilon} \varphi\left(y_{2}\right)\right|^{2}}{\left|y_{1}-y_{2}\right|^{1+2 s}} d y \\
& \leq\|\varphi\|_{L^{2}\left(I_{\varepsilon}\right)}^{2}+\|\varphi\|_{H^{s}\left(I_{\varepsilon}\right)}^{2}+I_{1}+I_{2}+I_{3},
\end{aligned}
$$

where

$$
\begin{gathered}
A=\left\{g_{\varepsilon}\left(x_{1}\right)<y_{1}<g_{1}\right\} \times\left\{g_{\varepsilon}\left(x_{1}\right)<y_{2}<g_{1}\right\}, \\
B=\left\{g_{\varepsilon}\left(x_{1}\right)<y_{1}<g_{1}\right\} \times\left\{0<y_{2}<g_{\varepsilon}\left(x_{1}\right)\right\}, \\
C=\left\{0<y_{1}<g_{\varepsilon}\left(x_{1}\right)\right\} \times\left\{g_{\varepsilon}\left(x_{1}\right)<y_{2}<g_{1}\right\} .
\end{gathered}
$$

We analyze each integral separately. If we change variables as $z_{i}=2 g_{\varepsilon}\left(x_{1}\right)-y_{i}, i=1,2$, we get:

$$
\begin{aligned}
\iint_{A} \frac{\left|P_{\varepsilon} \varphi\left(y_{1}\right)-P_{\varepsilon} \varphi\left(y_{2}\right)\right|^{2}}{\left|y_{1}-y_{2}\right|^{1+2 s}} d y & =\int_{g_{\varepsilon}\left(x_{1}\right)}^{g_{1}} \int_{g_{\varepsilon}\left(x_{1}\right)}^{g_{1}} \frac{\left|\varphi\left(2 g_{\varepsilon}\left(x_{1}\right)-y_{1}\right)-\varphi\left(2 g_{\varepsilon}\left(x_{1}\right)-y_{2}\right)\right|^{2}}{\left|y_{1}-y_{2}\right|^{1+2 s}} d y \\
& \leq 2 \iint_{I_{\varepsilon} \times I_{\varepsilon}} \frac{\left|\varphi\left(z_{1}\right)-\varphi\left(z_{2}\right)\right|^{2}}{\left|z_{1}-z_{2}\right|^{1+2 s}} d z .
\end{aligned}
$$

On the other hand, if $\left(y_{1}, y_{2}\right) \in B$, then $y_{2}<g_{\varepsilon}\left(x_{1}\right)$, and if we call $z_{1}=2 g_{\varepsilon}\left(x_{1}\right)-y_{1}$

$$
\begin{aligned}
\iint_{B} \frac{\left|P_{\varepsilon} \varphi\left(y_{1}\right)-P_{\varepsilon} \varphi\left(y_{2}\right)\right|^{2}}{\left|y_{1}-y_{2}\right|^{1+2 s}} d y & =\int_{g_{\varepsilon}\left(x_{1}\right)}^{g_{1}} \int_{0}^{g_{\varepsilon}\left(x_{1}\right)} \frac{\left|\varphi\left(2 g_{\varepsilon}\left(x_{1}\right)-y_{1}\right)-\varphi\left(y_{2}\right)\right|^{2}}{\left|y_{1}-y_{2}\right|^{1+2 s}} d y \\
& =\iint_{I_{\varepsilon} \times I_{\varepsilon}} \frac{\left|\varphi\left(z_{1}\right)-\varphi\left(y_{2}\right)\right|^{2}}{\left|2 g_{\varepsilon}\left(x_{1}\right)-z_{1}-y_{2}\right|^{1+2 s}} d y_{2} d z_{1} \\
& \leq 3 \iint_{I_{\varepsilon} \times I_{\varepsilon}} \frac{\left|\varphi\left(z_{1}\right)-\varphi\left(y_{2}\right)\right|^{2}}{\left|z_{1}-y_{2}\right|^{1+2 s}} d y_{2} d z_{1}
\end{aligned}
$$

since

$$
\begin{aligned}
\left|z_{1}-y_{2}\right| & =\left|2 g_{\varepsilon}\left(x_{1}\right)-y_{1}-y_{2}\right|=\left|y_{2}+y_{1}-2 g_{\varepsilon}\left(x_{1}\right)\right| \\
& \leq\left|y_{2}-y_{1}\right|+2\left|y_{1}-g_{\varepsilon}\left(x_{1}\right)\right| \leq 3\left|y_{1}-y_{2}\right|=3\left|2 g_{\varepsilon}\left(x_{1}\right)-z_{1}-y_{2}\right| .
\end{aligned}
$$

Analogously, we can show that

$$
\iint_{C} \frac{\left|P_{\varepsilon} \varphi\left(y_{1}\right)-P_{\varepsilon} \varphi\left(y_{2}\right)\right|^{2}}{\left|y_{1}-y_{2}\right|^{1+2 s}} d y \leq 3 \iint_{I_{\varepsilon} \times I_{\varepsilon}} \frac{\left|\varphi\left(y_{1}\right)-\varphi\left(z_{2}\right)\right|^{2}}{\left|y_{1}-z_{2}\right|^{1+2 s}} d z_{2} d y_{1}
$$

proving

$$
\left\|P_{\varepsilon} \varphi\right\|_{H^{s}(I)} \leq C\|\varphi\|_{H^{s}\left(I_{\varepsilon}\right)} .
$$

Now if $g_{1}>2 g_{0}$, we first extend the initial function $\varphi$ in the direction of negative $y$ and then construct $P_{\varepsilon}$ in an analogous way to the previous one. In fact, if $\varphi_{0}$ is defined in $I_{\varepsilon}$, we can extend it to $\left\{y \in \mathbb{R} ;-g_{0}<\right.$ $\left.y<g_{\varepsilon}\left(x_{1}\right)\right\}$ as

$$
\varphi_{1}(y)= \begin{cases}\varphi_{0}(y), & \text { if } 0<y<g_{\varepsilon}\left(x_{1}\right) \\ \varphi_{0}(-y), & \text { if }-g_{0}<y \leq 0\end{cases}
$$

Iteratively, we can take

$$
\varphi_{n}(y)=\left\{\begin{array}{ll}
\varphi_{n-1}(y), & \text { if }-(n-1) g_{0}<y<g_{\varepsilon}\left(x_{1}\right) \\
\varphi_{n-1}\left(-y-2(n-1) g_{0}\right), & \text { if }-n g_{0}<x_{2} \leq-(n-1) g_{0}
\end{array} .\right.
$$

Thus, given $\varphi_{0} \in H^{1}\left(I_{\varepsilon}\right)$, there is $n$ sufficiently large such that $n g_{0}>g_{1}$, and then, we can define $P_{\varepsilon}$ as

$$
\left(P_{\varepsilon} \varphi_{0}\right)(y)=\left\{\begin{array}{ll}
\varphi_{n}(y), & \text { if } y \in I_{\varepsilon} \\
\varphi_{n}\left(2 g_{\varepsilon}\left(x_{1}\right)-y\right), & \text { if } y \in I \backslash I_{\varepsilon}
\end{array} .\right.
$$


It is not difficult to see that $P_{\varepsilon}$ is well defined. Besides using $n g_{0}>g_{1}$, if $I_{\varepsilon}^{n}=\left(-n g_{0}, g_{1}\right)$, we have

$$
\begin{aligned}
& \left\|P_{\varepsilon} \varphi_{0}\right\|_{L^{2}(I)}^{2}=\int_{I}\left|P_{\varepsilon} \varphi_{0}\right|^{2}=\int_{I_{\varepsilon}}\left|P_{\varepsilon} \varphi_{0}\right|^{2}+\int_{I \backslash I_{\varepsilon}}\left|P_{\varepsilon} \varphi_{0}\right|^{2} \\
& \quad=\int_{I_{\varepsilon}}\left|\varphi_{n}\right|^{p}+\int_{g_{\varepsilon}\left(x_{1}\right)}^{g_{1}}\left|\varphi_{n}\left(x_{1}, 2 g_{\varepsilon}\left(x_{1}\right)-y\right)\right|^{2} d y \leq \int_{I_{\varepsilon}}\left|\varphi_{n}\right|^{2}+\int_{2 g_{\varepsilon}(x)-g_{1}}^{g_{\varepsilon}\left(x_{1}\right)}\left|\varphi_{n}(y)\right|^{2} d y \\
& \quad \leq 2\left\|\varphi_{n}\right\|_{L^{2}\left(I_{\varepsilon}^{n}\right)}^{2} \leq 2(n+1)\left\|\varphi_{0}\right\|_{L^{2}\left(I_{\varepsilon}\right)}^{2} .
\end{aligned}
$$

In a similar way, we can perform the same estimate in $H^{s}(I)$ and $H^{1}(I)$, obtaining a extension operator from $I_{\varepsilon}$ into the interval $I$. Finally, we can set $P$ to the whole real line. Indeed, for $u \in H^{1}\left(I_{\varepsilon}\right)$, let $\psi \in C_{c}^{\infty}(\mathbb{R})$ such that $I \subset \operatorname{supp}(\psi)$, with $\psi=1$ in $I$ and $\psi=0$ in $\mathbb{R} \backslash\left(-g_{0}, g_{1}+g_{0}\right)$. Then we set

$$
P u=\psi P_{\varepsilon}(u)
$$

completing the proof.

Now we state some properties of Lebesgue and Sobolev-Bochner generalized spaces that we will be needed in the analysis below.

Proposition 3.5. Let $I_{\varepsilon}=\left(0, g_{\varepsilon}\left(x_{1}\right)\right)$, with $\varepsilon>0, x_{1} \in(0,1)$ and $0<s<1$ fixed. Then there exist $C_{1}$, $C_{2}>0$ independent of $\varepsilon$ such that

$$
C_{1}\|u\|_{H^{s}\left(I_{\varepsilon}\right)} \leq\|u\|_{H_{[]}^{s}\left(I_{\varepsilon}\right)} \leq C_{2}\|u\|_{H^{s}\left(I_{\varepsilon}\right)}, \forall u \in H^{s}\left(I_{\varepsilon}\right)
$$

where $H_{[]}^{s}\left(I_{\varepsilon}\right)$ is the complex interpolation space

$$
H_{[]}^{s}\left(I_{\varepsilon}\right)=\left[L^{2}\left(I_{\varepsilon}\right), H^{1}\left(I_{\varepsilon}\right)\right]_{s}, \text { for } 0<s<1 .
$$

Proof. Using that there exists a continuous linear extension operator $P: L^{2}\left(I_{\varepsilon}\right) \rightarrow L^{2}(\mathbb{R})$ given by Lemma 3.4 if we define the space $\bar{H}^{s}\left(I_{\varepsilon}\right)=\left\{v_{\left.\right|_{\varepsilon}} ; v \in H^{s}(\mathbb{R})\right\}$, then for all $u \in H^{s}\left(I_{\varepsilon}\right)$

$$
K^{-1}\|u\|_{H^{s}\left(I_{\varepsilon}\right)} \leq\|u\|_{\bar{H}^{s}\left(I_{\varepsilon}\right)} \leq K \lambda_{s}\|u\|_{H^{s}\left(I_{\varepsilon}\right)}
$$

where $K>0$ and $\|P\|_{\mathcal{L}\left(H^{s}\left(I_{\varepsilon}\right), H^{s}(\mathbb{R})\right)} \leq \lambda_{s}$ are independent of $\varepsilon$, with same notation from Lemma 3.4 . Indeed, if $u \in \bar{H}^{s}\left(I_{\varepsilon}\right)$, there is $U \in H^{s}(\mathbb{R})$ such that $u=U_{I_{\varepsilon}}$ and $\|u\|_{\bar{H}^{s}\left(I_{\varepsilon}\right)}=\|U\|_{H(\mathbb{R})}$. It follows that

$$
\|u\|_{H^{s}\left(I_{\varepsilon}\right)} \leq\|U\|_{H^{s}(\mathbb{R})}=\|u\|_{\bar{H}^{s}\left(I_{\varepsilon}\right)} .
$$

Reciprocally, if $u \in H^{s}\left(I_{\varepsilon}\right)$, then $u=\left.U\right|_{I_{\varepsilon}}$ for $U=P u$. It follows that $u \in \bar{H}^{s}\left(I_{\varepsilon}\right)$, with

$$
\|u\|_{\bar{H}^{s}\left(I_{\varepsilon}\right)} \leq\|U\|_{H^{s}(\mathbb{R})}=\|P u\|_{H^{s}(\mathbb{R})} \leq \lambda_{s}\|u\|_{H^{s}\left(I_{\varepsilon}\right)} .
$$

Analogously, by [51, Lemma 4.2] and Lemma 3.4, we have

$$
\lambda_{0}^{s-1} \lambda_{1}^{-s}\|u\|_{\bar{H}^{s}\left(I_{\varepsilon}\right)} \leq\|u\|_{H_{[]}^{\theta}\left(I_{\varepsilon}\right)} \leq\|u\|_{\bar{H}^{s}\left(I_{\varepsilon}\right)} .
$$

Then

$$
\begin{aligned}
\lambda_{0}^{s-1} \lambda_{1}^{-s} K^{-1}\|u\|_{H^{s}\left(I_{\varepsilon}\right)} & \leq \lambda_{0}^{s-1} \lambda_{1}^{-s}\|v\|_{\bar{H}^{s}\left(I_{\varepsilon}\right)} \leq\|u\|_{H_{[]}^{\theta}\left(I_{\varepsilon}\right)} \\
& \leq\|u\|_{\bar{H}^{s}\left(I_{\varepsilon}\right)} \leq K \lambda_{s}\|u\|_{H^{s}\left(I_{\varepsilon}\right)}
\end{aligned}
$$

proving the result for $C_{1}=\lambda_{0}^{s-1} \lambda_{1}^{-s} K^{-1}$ and $C_{2}=K \lambda_{s}$, with $0<s<1$.

Proposition 3.6. For each $\varepsilon>0, H^{1}\left(\Omega_{\varepsilon}\right) \hookrightarrow L^{2}\left(0,1 ; H^{s}\left(0, g_{\varepsilon}\left(x_{1}\right)\right)\right)$ for all $0 \leq s \leq 1$, with embedding constant independent of $\varepsilon$. Moreover, if $0<s<1$, the embedding is compact.

Proof. For each $x_{1} \in(0,1)$ and $\varepsilon>0$, we have by Proposition 3.5 and properties of interpolation spaces that

$$
\left\|u\left(x_{1}, \cdot\right)\right\|_{H^{s}\left(0, g_{\varepsilon}\left(x_{1}\right)\right)} \leq C\left\|u\left(x_{1}, \cdot\right)\right\|_{H_{[]}^{s}\left(0, g_{\varepsilon}\left(x_{1}\right)\right)} \leq C\left\|u\left(x_{1}, \cdot\right)\right\|_{L^{2}\left(0, g_{\varepsilon}\left(x_{1}\right)\right)}^{1-s}\left\|u\left(x_{1}, \cdot\right)\right\|_{H^{1}\left(0, g_{\varepsilon}\left(x_{1}\right)\right)}^{s}
$$

where $C>0$ is independent of $\varepsilon>0$ and $x_{1} \in(0,1)$. It follows that

$$
\left\|u\left(x_{1}, \cdot\right)\right\|_{H^{s}\left(0, g_{\varepsilon}\left(x_{1}\right)\right)} \leq C\left\|u\left(x_{1}, \cdot\right)\right\|_{H^{s}\left(0, g_{\varepsilon}\left(x_{1}\right)\right)}^{1-s}\left\|u\left(x_{1}, \cdot\right)\right\|_{H^{1}\left(0, g_{\varepsilon}\left(x_{1}\right)\right)}^{s},
$$


and then

$$
\left\|u\left(x_{1}, \cdot\right)\right\|_{H^{s}\left(0, g_{\varepsilon}\left(x_{1}\right)\right)}^{s} \leq C\left\|u\left(x_{1}, \cdot\right)\right\|_{H^{1}\left(0, g_{\varepsilon}\left(x_{1}\right)\right)}^{s} .
$$

Consequently, if we integrate in $x_{1} \in(0,1)$

$$
\begin{aligned}
\|u\|_{L^{2}\left(0,1 ; H^{s}\left(0, g_{\varepsilon}\left(x_{1}\right)\right)\right)}^{2} & =\int_{0}^{1}\left\|u\left(x_{1}, \cdot\right)\right\|_{H^{s}\left(0, g_{\varepsilon}\left(x_{1}\right)\right)}^{2} d x_{1} \leq C \int_{0}^{1}\left\|u\left(x_{1}, \cdot\right)\right\|_{H^{1}\left(0, g_{\varepsilon}\left(x_{1}\right)\right)}^{2} d x_{1} \\
& =C\|u\|_{L^{2}\left(0,1 ; H^{1}\left(0, g_{\varepsilon}\left(x_{1}\right)\right)\right)}^{2} \leq C\|u\|_{H^{1}\left(\Omega_{\varepsilon}\right)}^{2},
\end{aligned}
$$

concluding the first statement. To prove the last one, let us consider $Q=(0,1)^{2}$ and the function

$$
\phi_{\varepsilon}: Q \rightarrow \Omega_{\varepsilon}:(x, y) \mapsto\left(x_{1}, x_{2}\right)=\phi_{\varepsilon}(x, y):=\left(x, y g_{\varepsilon}(x)\right) .
$$

Consequently, we can set

$$
\begin{aligned}
\Phi_{\varepsilon}: H^{1}\left(\Omega_{\varepsilon}\right) & \rightarrow H^{1}(Q) \\
u & \mapsto \Phi_{\varepsilon}(u):=u \circ \phi_{\varepsilon}
\end{aligned}
$$

and

$$
\begin{gathered}
\Psi_{\varepsilon}: L^{2}\left(0,1 ; H^{s}(0,1)\right) \rightarrow L^{2}\left(0,1 ; H^{s}\left(0, g_{\varepsilon}\left(x_{1}\right)\right)\right) \\
u \mapsto \Psi_{\varepsilon}(u):=u \circ \phi_{\varepsilon}^{-1} .
\end{gathered}
$$

It is not difficult to see that $\Phi$ and $\Psi$ are continuous and satisfy

$$
\left\|\Phi_{\varepsilon}(u)\right\|_{H^{1}(Q)} \leq C_{1}\|u\|_{H^{1}\left(\Omega_{\varepsilon}\right)} \quad \text { and } \quad\left\|\Psi_{\varepsilon}(u)\right\|_{L^{2}\left(0,1 ; H^{s}\left(0, g_{\varepsilon}\left(x_{1}\right)\right)\right)} \leq C_{2}\|u\|_{L^{2}\left(0,1 ; H^{s}(0,1)\right)}
$$

for every $0<s<1$, and constants $C_{1}, C_{2}>0$ independents of $\varepsilon$.

Hence, we can use [41, Proposition 3.57] to obtain that the inclusion

$$
H^{1}(Q) \hookrightarrow L^{2}\left(0,1 ; H^{s}(0,1)\right)
$$

is compact. Thus, we have the following chain

$$
H^{1}\left(\Omega_{\varepsilon}\right) \stackrel{\Phi_{\varepsilon}}{\hookrightarrow} H^{1}(Q) \hookrightarrow L^{2}\left(0,1 ; H^{s}(0,1)\right) \stackrel{\Psi_{\varepsilon}}{\hookrightarrow} L^{2}\left(0,1 ; H^{s}\left(0, g_{\varepsilon}\left(x_{1}\right)\right)\right),
$$

that implies the compact immersion.

3.2. Concentrated integrals. Finally, we consider here what we call concentrated integrals.

Theorem 3.7. For $\varepsilon_{0}>0$ sufficiently small, there is a constant $C>0$, independent of $\varepsilon \in\left(0, \varepsilon_{0}\right)$ and $u^{\varepsilon} \in H^{1}\left(\Omega_{\varepsilon}\right)$, such that, for all $1 / 2<s \leq 1$,

$$
\frac{1}{\varepsilon} \int_{\theta_{\varepsilon}}\left|u^{\varepsilon}\right|^{q} \leq C\left\|u^{\varepsilon}\right\|_{L^{q}\left(0,1 ; H^{s}\left(0, g_{\varepsilon}\left(x_{1}\right)\right)\right)}^{q}, \quad \forall q \geq 1
$$

and

$$
\frac{1}{\varepsilon} \int_{\theta_{\varepsilon}}\left|u^{\varepsilon}\right|^{2} \leq C\left(\left\|u^{\varepsilon}\right\|_{H^{s}\left(\Omega_{\varepsilon}\right)}^{2}+\left\|\frac{\partial u^{\varepsilon}}{\partial x_{2}}\right\|_{L^{2}\left(\Omega_{\varepsilon}\right)}^{2}\right) .
$$

In particular,

$$
\frac{1}{\varepsilon} \int_{\theta_{\varepsilon}}\left|u^{\varepsilon}\right|^{2} \leq C\left\|u^{\varepsilon}\right\|_{H^{1}\left(\Omega_{\varepsilon}\right)}^{2} .
$$

Proof. Take $u \in H^{1}\left(\Omega_{\varepsilon}\right)$. In a.e. $x_{1} \in(0,1)$, we have $u\left(x_{1}, \cdot\right) \in H^{1}\left(0, g_{\varepsilon}\left(x_{1}\right)\right)$. Define

$$
z^{*}:=g_{0}-\varepsilon_{0} h_{1} \text { and } z^{\varepsilon}:=g_{\varepsilon}\left(x_{1}\right)-\varepsilon h_{\varepsilon}\left(x_{1}\right)
$$

for $\varepsilon_{0}>0$ sufficiently small in such way that, for all $\varepsilon<\varepsilon_{0}$, we have

$$
\left[z^{\varepsilon}-z^{*}, z^{\varepsilon}\right] \subset\left[0, g_{\varepsilon}\left(x_{1}\right)\right] .
$$




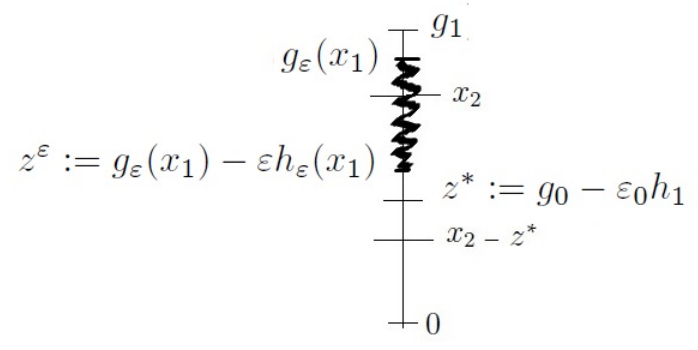

Figure 3. Fixed $x_{1} \in(0,1)$ and $\varepsilon>0$, we get this fiber to the oscillatory domain for $\varepsilon<\varepsilon_{0}$.

Since $\left(g_{\varepsilon}\left(x_{1}\right)-\varepsilon h_{\varepsilon}\left(x_{1}\right)\right)<x_{2}<g_{\varepsilon}\left(x_{1}\right)$ and $1 / 2<s \leq 1$, it follows from [48, Theorem 1.5.1.3] for $n=1$ that exists $K>0$ independent of $\varepsilon>0$ such that

$$
\left|u\left(x_{1}, x_{2}\right)\right| \leq K\left\|u\left(x_{1}, \cdot\right)\right\|_{H^{s}\left(x_{2}-z^{*}, x_{2}\right)} \leq K\left\|u\left(x_{1}, \cdot\right)\right\|_{H^{s}\left(0, g_{\varepsilon}\left(x_{1}\right)\right)} .
$$

Indeed, the interval where we are applying the result is fixed and independent of the parameters $\varepsilon$ and $x_{1}$.

Hence,

$$
\begin{aligned}
\frac{1}{\varepsilon} \int_{\theta_{\varepsilon}}|u|^{q} & =\int_{0}^{1} \frac{1}{\varepsilon} \int_{g_{\varepsilon}\left(x_{1}\right)-\varepsilon h_{\varepsilon}\left(x_{1}\right)}^{g_{\varepsilon}\left(x_{1}\right)}\left|u\left(x_{1}, x_{2}\right)\right|^{q} d x_{2} d x_{1} \\
& \leq \int_{0}^{1} \frac{1}{\varepsilon} \int_{g_{\varepsilon}\left(x_{1}\right)-\varepsilon h_{\varepsilon}\left(x_{1}\right)}^{g_{\varepsilon}\left(x_{1}\right)} K^{q}\left\|u\left(x_{1}, \cdot\right)\right\|_{H^{s}\left(0, g_{\varepsilon}\left(x_{1}\right)\right)}^{q} d x_{2} d x_{1} \\
& \leq K^{q} h_{1} \int_{0}^{1}\left\|u\left(x_{1}, \cdot\right)\right\|_{H^{s}\left(0, g_{\varepsilon}\left(x_{1}\right)\right)}^{q} d x_{1}=C_{1}\|u\|_{L^{q}\left(0,1 ; H^{s}\left(0, g_{\varepsilon}\left(x_{1}\right)\right)\right)}^{q},
\end{aligned}
$$

where $C_{2}$ is independent of $\varepsilon$, proving (3.1).

If $q=2$, due to Proposition 3.6 and previous inequality, we get

$$
\frac{1}{\varepsilon} \int_{\theta_{\varepsilon}}|u|^{2} \leq C_{1}\|u\|_{L^{2}\left(0,1 ; H^{s}\left(0, g_{\varepsilon}\left(x_{1}\right)\right)\right)}^{2} \leq C_{1} C_{2}\|u\|_{H^{1}\left(\Omega_{\varepsilon}\right)}^{2}
$$

proving 3.3 .

Now, let us prove 3.2 . Here we use that $C^{\infty}\left(\Omega_{\varepsilon}\right)$ is dense in $H^{1}\left(\Omega_{\varepsilon}\right)$ (see [48, Theorem 1.4.2.2]). Let $u \in C^{\infty}\left(\Omega_{\varepsilon}\right)$ and fixed $x_{1} \in(0,1)$. By Fundamental Theorem of Calculus, we have

$$
u\left(x_{1}, x_{2}\right)=u\left(x_{1}, 0\right)+\int_{0}^{x_{2}} \frac{\partial u}{\partial x_{2}}\left(x_{1}, s\right) d s .
$$

Then

$$
\begin{aligned}
\left|u\left(x_{1}, x_{2}\right)\right|^{2} & \leq 2\left|u\left(x_{1}, 0\right)\right|^{2}+2\left[\left(\int_{0}^{x_{2}}\left|\frac{\partial u}{\partial x_{2}}\left(x_{1}, s\right)\right|^{2} d s\right)^{1 / 2}\left(\int_{0}^{x_{2}} 1^{2} d s\right)^{1 / 2}\right]^{2} \\
& \leq 2\left|u\left(x_{1}, 0\right)\right|^{2}+2 g_{\varepsilon}\left(x_{1}\right) \int_{0}^{x_{2}}\left|\frac{\partial u}{\partial x_{2}}\left(x_{1}, s\right)\right|^{2} d s
\end{aligned}
$$

Consequently,

$$
\begin{aligned}
\int_{g_{\varepsilon}\left(x_{1}\right)-\varepsilon h\left(x_{1}, \varepsilon\right)}^{g_{\varepsilon}\left(x_{1}\right)}\left|u\left(x_{1}, x_{2}\right)\right|^{2} d x_{2} \leq 2 \int_{g_{\varepsilon}\left(x_{1}\right)-\varepsilon h_{\varepsilon}\left(x_{1}\right)}^{g_{\varepsilon}\left(x_{1}\right)}\left|u\left(x_{1}, 0\right)\right|^{2} d x_{2} \\
+2 g_{\varepsilon}\left(x_{1}\right) \int_{g_{\varepsilon}\left(x_{1}\right)-\varepsilon h_{\varepsilon}\left(x_{1}\right)}^{g_{\varepsilon}\left(x_{1}\right)}\left(\int_{0}^{x_{2}}\left|\frac{\partial u}{\partial x_{2}}\left(x_{1}, s\right)\right|^{2} d s\right) d x_{2}
\end{aligned}
$$




$$
\leq 2 \varepsilon h_{1}\left|u\left(x_{1}, 0\right)\right|^{2}+2 g_{1} \varepsilon h_{1} \int_{0}^{g_{\varepsilon}\left(x_{1}\right)}\left|\frac{\partial u}{\partial x_{2}}\left(x_{1}, x_{2}\right)\right|^{2} d x_{2} .
$$

Hence, if $\gamma(u)$ is the trace of $u$ given by [48, Theorem 1.5.1.3], we get

$$
\begin{aligned}
\frac{1}{\varepsilon} \int_{\theta_{\varepsilon}}|u|^{2} & =\frac{1}{\varepsilon} \int_{0}^{1} \int_{g_{\varepsilon}\left(x_{1}\right)-\varepsilon h_{\varepsilon}\left(x_{1}\right)}^{g_{\varepsilon}\left(x_{1}\right)}\left|u\left(x_{1}, x_{2}\right)\right|^{2} d x_{2} d x_{1} \\
& \leq 2 h_{1} \int_{0}^{1}\left|u\left(x_{1}, 0\right)\right|^{2} d x_{1}+2 g_{1} h_{1} \int_{0}^{1} \int_{0}^{g_{\varepsilon}\left(x_{1}\right)}\left|\frac{\partial u}{\partial x_{2}}\left(x_{1}, x_{2}\right)\right|^{2} d x_{2} d x_{1} \\
& \leq 2 h_{1}\left(\|\gamma(u)\|_{L^{2}(0,1)}^{2}+g_{1}\left\|\frac{\partial u}{\partial x_{2}}\right\|_{L^{2}\left(\Omega_{\varepsilon}\right)}^{2}\right) .
\end{aligned}
$$

On the other hand, if $\Omega_{0}=(0,1) \times\left(0, g_{0}\right)$, we have $\Omega_{0} \subset \Omega_{\varepsilon}$, and there exists a constant $c>0$ such that $\|\gamma(u)\|_{L^{2}(0,1)} \leq c\|u\|_{H^{s}\left(\Omega_{0}\right)}$ for all $1 / 2<s \leq 1$. Then, due to the previous inequality,

$$
\frac{1}{\varepsilon} \int_{\theta_{\varepsilon}}|u|^{2} \leq 2 h_{1}\left(c\|u\|_{H^{s}\left(\Omega_{0}\right)}^{2}+g_{1}\left\|\frac{\partial u}{\partial x_{2}}\right\|_{L^{2}\left(\Omega_{\varepsilon}\right)}^{2}\right) \leq C_{1}\left(\|u\|_{H^{s}\left(\Omega_{\varepsilon}\right)}^{2}+\left\|\frac{\partial u}{\partial x_{2}}\right\|_{L^{2}\left(\Omega_{\varepsilon}\right)}^{2}\right)
$$

with $C_{1}$ independent of $\varepsilon$.

\section{Nonlinearities}

In this section, we show some properties to a class of nonlinear maps defined in Sobolev-Bochner spaces. Such applications will define the nonlinearity of our elliptic problems.

Consider the Sobolev-Bochner spaces

$$
X_{\varepsilon}=L^{2}\left(0,1 ; H^{s}\left(0, g\left(x_{1}, \varepsilon\right)\right)\right), \text { and their dual } X_{\varepsilon}^{\prime}=L^{2}\left(0,1 ;\left\{H^{s}\left(0, g\left(x_{1}, \varepsilon\right)\right)\right\}^{\prime}\right),
$$

for $1 / 2<s<1$, and define

$$
\begin{aligned}
F_{\varepsilon}: X_{\varepsilon} & \rightarrow X_{\varepsilon}^{\prime} \\
u & \mapsto F_{\varepsilon}(u): X_{\varepsilon} \rightarrow \mathbb{R} \\
v & \mapsto\left\langle F_{\varepsilon}(u), v\right\rangle=\frac{1}{\varepsilon} \int_{\theta_{\varepsilon}} f(u) v
\end{aligned}
$$

where $f \in C^{2}(\mathbb{R})$ is a bounded function with bounded derivatives (see Remark 2.6). Thus, we have:

Proposition 4.1. The function $F_{\varepsilon}$ defined in $(4.2)$ satisfies, with constants independents of $\varepsilon$ :

(a) there is $K>0$ such that

$$
\sup _{u^{\varepsilon} \in X_{\varepsilon}}\left\|F_{\varepsilon}\left(u^{\varepsilon}\right)\right\|_{X_{\varepsilon}^{\prime}} \leq K
$$

(b) $F_{\varepsilon}$ is Lipschitz and, therefore, is continuous; in other words there is $L>0$ such that

$$
\left\|F_{\varepsilon}\left(u_{1}^{\varepsilon}\right)-F_{\varepsilon}\left(u_{2}^{\varepsilon}\right)\right\|_{X_{\varepsilon}^{\prime}} \leq L\left\|u_{1}^{\varepsilon}-u_{2}^{\varepsilon}\right\|_{X_{\varepsilon}}, \forall u_{1}, u_{2} \in X_{\varepsilon}
$$

Proof. (a) For $u^{\varepsilon} \in X_{\varepsilon}$,

$$
\left\|F_{\varepsilon}\left(u^{\varepsilon}\right)\right\|_{X_{\varepsilon}^{\prime}}=\sup _{\left\|v^{\varepsilon}\right\|_{X_{\varepsilon}}=1}\left|\left\langle F_{\varepsilon}\left(u^{\varepsilon}\right), v^{\varepsilon}\right\rangle\right| .
$$

So, if $v^{\varepsilon} \in X_{\varepsilon}$, using Theorem 3.7 we have

$$
\left|\left\langle F_{\varepsilon}\left(u^{\varepsilon}\right), v^{\varepsilon}\right\rangle\right| \leq \frac{1}{\varepsilon} \int_{\theta_{\varepsilon}}\left|f\left(u^{\varepsilon}\right) v^{\varepsilon}\right| \leq\left(\frac{1}{\varepsilon} \int_{\theta_{\varepsilon}}\left|f\left(u^{\varepsilon}\right)\right|^{2}\right)^{1 / 2}\left(\frac{1}{\varepsilon} \int_{\theta_{\varepsilon}}\left|v^{\varepsilon}\right|^{2}\right)^{1 / 2} \leq\|f\|_{\infty} h_{1}^{1 / 2} C\left\|v^{\varepsilon}\right\|_{X_{\varepsilon}} .
$$

Therefore

$$
\sup _{u^{\varepsilon} \in X_{\varepsilon}}\left\|F_{\varepsilon}\left(u^{\varepsilon}\right)\right\|_{X_{\varepsilon}^{\prime}} \leq\|f\|_{\infty} h_{1}^{1 / 2} C \leq K
$$


(b) Indeed, if $u_{1}^{\varepsilon}, u_{2}^{\varepsilon} \in X_{\varepsilon}$ then

$$
\left\|F_{\varepsilon}\left(u_{1}^{\varepsilon}\right)-F_{\varepsilon}\left(u_{2}^{\varepsilon}\right)\right\|_{X_{\varepsilon}^{\prime}}=\sup _{\left\|v^{\varepsilon}\right\| X_{\varepsilon}=1}\left|\left\langle F_{\varepsilon}\left(u_{1}^{\varepsilon}\right), v^{\varepsilon}\right\rangle-\left\langle F_{\varepsilon}\left(u_{2}^{\varepsilon}\right), v^{\varepsilon}\right\rangle\right|
$$

Using Theorem 3.7 .

$$
\begin{aligned}
\mid\left\langle F_{\varepsilon}\left(u_{1}^{\varepsilon}\right), v^{\varepsilon}\right\rangle-\langle & \left.F_{\varepsilon}\left(u_{2}^{\varepsilon}\right), v^{\varepsilon}\right\rangle|=|\left\langle F_{\varepsilon}\left(u_{1}^{\varepsilon}\right)-F_{\varepsilon}\left(u_{2}^{\varepsilon}\right), v^{\varepsilon}\right\rangle\left|\leq \frac{1}{\varepsilon} \int_{\theta_{\varepsilon}}\right|\left(f\left(u_{1}^{\varepsilon}\right)-f\left(u_{2}^{\varepsilon}\right)\right) v^{\varepsilon} \mid \\
& \leq\left(\frac{1}{\varepsilon} \int_{\theta_{\varepsilon}}\left|f\left(u_{1}^{\varepsilon}\right)-f\left(u_{2}^{\varepsilon}\right)\right|^{2}\right)^{1 / 2}\left(\frac{1}{\varepsilon} \int_{\theta_{\varepsilon}}\left|v^{\varepsilon}\right|^{2}\right)^{1 / 2} \\
& \leq\left\|f^{\prime}\right\|_{\infty} C^{2}\left\|u_{1}^{\varepsilon}-u_{2}^{\varepsilon}\right\|_{X_{\varepsilon}}\left\|v^{\varepsilon}\right\|_{X_{\varepsilon}}
\end{aligned}
$$

Thus

$$
\left\|F_{\varepsilon}\left(u_{1}^{\varepsilon}\right)-F_{\varepsilon}\left(u_{2}^{\varepsilon}\right)\right\|_{X_{\varepsilon}^{\prime}} \leq\left\|f^{\prime}\right\|_{\infty} C^{2}\left\|u_{1}^{\varepsilon}-u_{2}^{\varepsilon}\right\|_{X_{\varepsilon}}
$$

and, therefore, $F_{\varepsilon}$ is Lipschitz with constant independent of $\varepsilon$.

\section{UPPER AND LOWER SEMICONTINUITY}

In this section, we prove the main result passing to the limit in problem (2.4). First, we write equations (2.2) and 2.4 in an abstract way. Next, we combine the results from the previous sections with those ones from [42, 45] concerned with compact convergence to obtain upper and lower semicontinuity to $\mathcal{E}_{\varepsilon, R}$ at $\varepsilon=0$.

5.1. Abstract setting and existence of solutions. In order to write problem (2.4) in an abstract way, we consider the linear operator

$$
\begin{aligned}
A_{\varepsilon}: D\left(A_{\varepsilon}\right) \subset L^{2}\left(\Omega_{\varepsilon}\right) \rightarrow L^{2}\left(\Omega_{\varepsilon}\right) \\
u^{\varepsilon} \longmapsto A_{\varepsilon} u^{\varepsilon}:=-\frac{\partial^{2} u^{\varepsilon}}{\partial x_{1}^{2}}-\frac{1}{\varepsilon^{2}} \frac{\partial^{2} u^{\varepsilon}}{\partial x_{2}^{2}}+u^{\varepsilon},
\end{aligned}
$$

with $D\left(A_{\varepsilon}\right):=\left\{u^{\varepsilon} \in H^{2}\left(\Omega_{\varepsilon}\right) ; \frac{\partial u^{\varepsilon}}{\partial x_{1}} N_{1}+\frac{1}{\varepsilon^{2}} \frac{\partial u^{\varepsilon}}{\partial x_{2}} N_{2}=0\right\}$.

Let $Z_{\varepsilon}^{0}=L^{2}\left(\Omega_{\varepsilon}\right), Z_{\varepsilon}^{1}=D\left(A_{\varepsilon}\right)$ and consider the scale of Hilbert spaces $Z_{\varepsilon}^{\alpha}$ constructed by complex interpolation between $Z_{\varepsilon}^{0}$ and $Z_{\varepsilon}^{1}$. In our context, such spaces isometrically coincide with the fractional power space $A_{\varepsilon}^{\alpha}$ of the operator $A_{\varepsilon}$ (see [49, Theorem 16.1]). Such scale can be extended to negative exponents taking $Z_{\varepsilon}^{-\alpha}=\left(Z_{\varepsilon}^{\alpha}\right)^{\prime}$ for $\alpha>0$. Notice that $Z_{\varepsilon}^{1 / 2}=H_{\varepsilon}^{1}\left(\Omega_{\varepsilon}\right)$ and $Z_{\varepsilon}^{-1 / 2}=\left(H_{\varepsilon}^{1}\left(\Omega_{\varepsilon}\right)\right)^{\prime}$ where $H_{\varepsilon}^{1}\left(\Omega_{\varepsilon}\right)$ is the space $H^{1}\left(\Omega_{\varepsilon}\right)$ endowed with the equivalent norm

$$
\left\|u^{\varepsilon}\right\|_{H_{\varepsilon}^{1}\left(\Omega_{\varepsilon}\right)}^{2}=\left\|u^{\varepsilon}\right\|_{L^{2}\left(\Omega_{\varepsilon}\right)}^{2}+\left\|\frac{\partial u^{\varepsilon}}{\partial x_{1}}\right\|_{L^{2}\left(\Omega_{\varepsilon}\right)}^{2}+\frac{1}{\varepsilon^{2}}\left\|\frac{\partial u^{\varepsilon}}{\partial x_{2}}\right\|_{L^{2}\left(\Omega_{\varepsilon}\right)}^{2} .
$$

Then, if we consider the realizations of $A_{\varepsilon}$ in this scale, we obtain $A_{\varepsilon,-1 / 2} \in \mathcal{L}\left(Z_{\varepsilon}^{1 / 2}, Z_{\varepsilon}^{-1 / 2}\right)$ with

$$
\left\langle A_{\varepsilon,-1 / 2} u^{\varepsilon}, \varphi^{\varepsilon}\right\rangle=\int_{\Omega_{\varepsilon}} \frac{\partial u^{\varepsilon}}{\partial x_{1}} \frac{\partial \varphi^{\varepsilon}}{\partial x_{1}}+\frac{1}{\varepsilon^{2}} \frac{\partial u^{\varepsilon}}{\partial x_{2}} \frac{\partial \varphi^{\varepsilon}}{\partial x_{2}}+u^{\varepsilon} \varphi^{\varepsilon}, \quad \forall \varphi^{\varepsilon} \in H^{1}\left(\Omega_{\varepsilon}\right) .
$$

With some abuse of notation, we identify all different realizations of this operator writing them as $A_{\varepsilon}$. Then problem 2.4 can be rewrite as

$$
A_{\varepsilon} u^{\varepsilon}=F_{\varepsilon}\left(u^{\varepsilon}\right)
$$

where the map $F_{\varepsilon}$ is given by

$$
\begin{aligned}
& F_{\varepsilon}: L^{2}\left(0,1 ; H^{s}\left(0, g_{\varepsilon}(x)\right)\right) \rightarrow L^{2}\left(0,1 ;\left\{H^{s}\left(0, g_{\varepsilon}(x)\right)\right\}^{\prime}\right) \\
& u^{\varepsilon} \mapsto F_{\varepsilon}\left(u^{\varepsilon}\right): L^{2}\left(0,1 ; H^{s}\left(0, g_{\varepsilon}(x)\right)\right) \rightarrow \mathbb{R} \\
& v^{\varepsilon} \mapsto\left\langle F_{\varepsilon}\left(u^{\varepsilon}\right), v^{\varepsilon}\right\rangle=\frac{1}{\varepsilon} \int_{\theta_{\varepsilon}} f\left(u^{\varepsilon}\right) v^{\varepsilon},
\end{aligned}
$$


with $1 / 2<s<1$.

Thus $u^{\varepsilon} \in H^{1}\left(\Omega_{\varepsilon}\right)$ is a solution of (5.1) if, and only if, $u^{\varepsilon}=A_{\varepsilon}^{-1} F_{\varepsilon}\left(u^{\varepsilon}\right)$. Then $u^{\varepsilon} \in H^{1}\left(\Omega_{\varepsilon}\right)$ must be a fixed point to $\left.A_{\varepsilon}^{-1} F_{\varepsilon}\right|_{H^{1}\left(\Omega_{\varepsilon}\right)}: H^{1}\left(\Omega_{\varepsilon}\right) \rightarrow H^{1}\left(\Omega_{\varepsilon}\right)$. The existence of such solutions follows from Schaefer Fixed Point Theorem [53, Section 9.2.2, Theorem 4].

In a similar way, we can analyze the limit problem given by $(2.2)$. We first consider $X_{0}=L^{2}(0,1)$ with the norm $\|u\|_{X_{0}}^{2}=\mu_{g}\|u\|_{L^{2}(0,1)}^{2}$ and, then, the linear operator

$$
\begin{aligned}
A_{0}: D\left(A_{0}\right) \subset X_{0} & \rightarrow X_{0} \\
u & \mapsto A_{0} u=-q_{0} u_{x x}+u,
\end{aligned}
$$

with $D\left(A_{0}\right)=\left\{u \in H^{2}(0,1) ; u^{\prime}(0)=u^{\prime}(1)=0\right\}$. Next, we introduce the fractional power spaces, and set the nonlinearity

$$
\begin{aligned}
F_{0}: X_{0} & \rightarrow L^{2}(0,1) \\
u & \mapsto F_{0}(u): L^{2}(0,1) \rightarrow \mathbb{R} \\
v & \mapsto\left\langle F_{0}(u), v\right\rangle=\int_{0}^{1} \mu_{h} f_{0}(u) d x,
\end{aligned}
$$

where $q_{0}$ and $f_{0}$ are given in 2.3 .

Consequently, the limit problem 2.2 can be rewritten as

$$
A_{0} u=F_{0}(u)
$$

and then, $u \in H^{1}(\Omega)$ is a solution of (5.2) if, and only if, $u=A_{0}^{-1} F_{0}(u)$. Thus, $u \in H^{1}(0,1)$ is a fixed point to $A_{0}^{-1} F_{0}: H^{1}(0,1) \rightarrow H^{1}(0,1)$. The existence of a solution also follows from Schauder's Fixed Point Theorem.

5.2. Extension operator. Now, we consider a continuous extension linear operator that will be useful in our situation. More precisely, from [21, Lemma 3.1] we have

Lemma 5.1. If $\Omega_{\varepsilon}=\left\{\left(x_{1}, x_{2}\right) ; 0<x_{1}<1,0<x_{2}<g_{\varepsilon}\left(x_{1}\right)\right\}$ and $\Omega=\left\{\left(x_{1}, x_{2}\right) ; 0<x_{1}<1,0<x_{2}<g_{1}\right\}$, then there exist a constant $K>0$, independent of $\varepsilon$ and $p$, and an extension operator

$$
P_{\varepsilon} \in \mathcal{L}\left(L^{p}\left(\Omega_{\varepsilon}\right), L^{p}(\Omega)\right) \cap \mathcal{L}\left(W^{1, p}\left(\Omega_{\varepsilon}\right), W^{1, p}(\Omega)\right) \cap \mathcal{L}\left(W_{\partial_{l}}^{1, p}\left(\Omega_{\varepsilon}\right), W_{\partial_{l}}^{1, p}(\Omega)\right)
$$

(where $W_{\partial_{l}}^{1, p}$ is the set of functions in $W^{1, p}$ that vanish in the domain's lateral boundary) such that

$$
\begin{gathered}
\left\|P_{\varepsilon} \varphi^{\varepsilon}\right\|_{L^{p}(\Omega)} \leq K\left\|\varphi^{\varepsilon}\right\|_{L^{p}\left(\Omega_{\varepsilon}\right)}, \\
\left\|\frac{\partial P_{\varepsilon} \varphi^{\varepsilon}}{\partial x_{1}}\right\|_{L^{p}(\Omega)} \leq K\left(\left\|\frac{\partial \varphi^{\varepsilon}}{\partial x_{1}}\right\|_{L^{p}\left(\Omega_{\varepsilon}\right)}+\eta(\varepsilon)\left\|\frac{\partial \varphi^{\varepsilon}}{\partial x_{2}}\right\|_{L^{p}\left(\Omega_{\varepsilon}\right)}\right), \\
\left\|\frac{\partial P_{\varepsilon} \varphi^{\varepsilon}}{\partial x_{2}}\right\|_{L^{p}(\Omega)} \leq K\left\|\frac{\partial \varphi^{\varepsilon}}{\partial x_{2}}\right\|_{L^{p}\left(\Omega_{\varepsilon}\right)},
\end{gathered}
$$

for all $\varphi^{\varepsilon} \in W^{1, p}\left(\Omega_{\varepsilon}\right)$, with $1 \leq p \leq \infty$ and $\eta(\varepsilon)=\sup \left|g_{\varepsilon}^{\prime}\left(x_{1}\right)\right|$.

This operator will play an important role in the convergence analysis since it extends the functions defined in the perturbed domain $\Omega_{\varepsilon}$ into the fixed one $\Omega$ in an appropriate way. One important property of this extension operator is the following.

Proposition 5.2. If $\|u\|_{H_{\varepsilon}^{1}\left(\Omega_{\varepsilon}\right)} \leq K$, with $K>0$ independent of $\varepsilon$, then $\left\|P_{\varepsilon} u\right\|_{H^{1}(\Omega)}$ is uniformly bounded and we can extract a subsequence (still denoted by $\varepsilon$ ) such that

$$
P_{\varepsilon} u^{\varepsilon} \rightarrow u_{0} \text { in } L^{2}(\Omega), \quad P_{\varepsilon} u^{\varepsilon} \rightarrow u_{0} \text { in } H^{1}(\Omega), \quad \frac{\partial P_{\varepsilon} u^{\varepsilon}}{\partial x_{2}} \rightarrow 0 \text { in } L^{2}(\Omega),
$$

for some $u_{0} \in H^{1}(0,1)$, where $P_{\varepsilon}$ is the extension operator from Lemma 5.1. In particular, if $1 / 2<s<1$,

$$
P_{\varepsilon} u^{\varepsilon} \rightarrow u_{0}, \quad \text { in } X=L^{2}\left(0,1 ; H^{s}\left(0, g_{1}\right)\right) \text {. }
$$


Proof. In fact, since $\left\|u^{\varepsilon}\right\|_{H_{\varepsilon}^{1}\left(\Omega_{\varepsilon}\right)} \leq C$ we have

Using Lemma 5.1 .

$$
\left\|u^{\varepsilon}\right\|_{L^{2}(\Omega)} \leq C, \quad\left\|\frac{\partial u^{\varepsilon}}{\partial x_{1}}\right\|_{L^{2}(\Omega)} \leq C \text { and }\left\|\frac{\partial u^{\varepsilon}}{\partial x_{2}}\right\|_{L^{2}(\Omega)} \leq C \varepsilon .
$$

$$
\begin{aligned}
\left\|P_{\varepsilon} u^{\varepsilon}\right\|_{H^{1}(\Omega)}^{2} & =\left\|P_{\varepsilon} u^{\varepsilon}\right\|_{L^{2}(\Omega)}^{2}+\left\|\frac{\partial P_{\varepsilon} u^{\varepsilon}}{\partial x_{1}}\right\|_{L^{2}(\Omega)}^{2}+\left\|\frac{\partial P_{\varepsilon} u^{\varepsilon}}{\partial x_{2}}\right\|_{L^{2}(\Omega)}^{2} \\
& \leq K\left[\left\|u^{\varepsilon}\right\|_{L^{2}\left(\Omega_{\varepsilon}\right)}^{2}+\left(\left\|\frac{\partial u^{\varepsilon}}{\partial x_{1}}\right\|_{L^{2}\left(\Omega_{\varepsilon}\right)}^{2}+\eta^{2}(\varepsilon)\left\|\frac{\partial u^{\varepsilon}}{\partial x_{2}}\right\|_{L^{2}\left(\Omega_{\varepsilon}\right)}^{2}\right)+\left\|\frac{\partial u^{\varepsilon}}{\partial x_{2}}\right\|_{L^{2}\left(\Omega_{\varepsilon}\right)}^{2}\right]
\end{aligned}
$$

Since $g$ has bounded derivative, it follows

$$
\eta(\varepsilon)=\frac{1}{\varepsilon} \sup \left|g^{\prime}\left(x_{1} / \varepsilon\right)\right| \leq \frac{C}{\varepsilon},
$$

and then, for $0<\varepsilon<1$ there is $M>0$ independent of $\varepsilon>0$ such that

$$
\left\|P_{\varepsilon} u^{\varepsilon}\right\|_{H^{1}(\Omega)} \leq \bar{C}\left\|u^{\varepsilon}\right\|_{H_{\varepsilon}^{1}\left(\Omega_{\varepsilon}\right)}^{2} \leq M \text {. }
$$

Consequently,

$$
\left\|P_{\varepsilon} u^{\varepsilon}\right\|_{L^{2}(\Omega)} \leq M,\left\|\frac{\partial P_{\varepsilon} u^{\varepsilon}}{\partial x_{1}}\right\|_{L^{2}(\Omega)} \leq M,\left\|\frac{\partial P_{\varepsilon} u^{\varepsilon}}{\partial x_{2}}\right\|_{L^{2}(\Omega)} \leq \varepsilon M .
$$

Thus, there exist a subsequence $P_{\varepsilon} u^{\varepsilon}$ and a function $u_{0} \in H^{1}(\Omega)$ such that

$$
P_{\varepsilon} u^{\varepsilon} \rightarrow u_{0} \text { in } L^{2}(\Omega), \quad P_{\varepsilon} u^{\varepsilon} \rightarrow u_{0} \text { in } H^{1}(\Omega), \quad \frac{\partial P_{\varepsilon} u^{\varepsilon}}{\partial x_{2}} \rightarrow 0 \text { in } L^{2}(\Omega) .
$$

Further, from Proposition 3.6, we have $P_{\varepsilon} u^{\varepsilon} \rightarrow u_{0}$ in $X=L^{2}\left(0,1 ; H^{s}\left(0, g_{1}\right)\right)$.

It follows that $u_{0}\left(x_{1}, x_{2}\right)=u_{0}\left(x_{1}\right)$ in $\Omega$, in particular,

$$
\frac{\partial u_{0}}{\partial x_{2}}\left(x_{1}, x_{2}\right)=0 \text { a.e. in } \Omega \text {. }
$$

Indeed, for all $\varphi \in C_{0}^{\infty}(\Omega)$, we have

$$
\int_{\Omega} \frac{\partial u_{0}}{\partial x_{2}} \varphi d x_{1} d x_{2}=-\int_{\Omega} u_{0} \frac{\partial \varphi}{\partial x_{2}} d x_{1} d x_{2}=-\lim _{\varepsilon \rightarrow 0} \int_{\Omega}\left(P_{\varepsilon} u^{\varepsilon}\right) \frac{\partial \varphi}{\partial x_{2}} d x_{1} d x_{2}=-\lim _{\varepsilon \rightarrow 0} \int_{\Omega} \frac{\partial P_{\varepsilon} u^{\varepsilon}}{\partial x_{2}} \varphi d x_{1} d x_{2}=0 .
$$

Furthermore, since $u_{0} \in H^{1}(\Omega)$ and $u_{0}(x, y)$ does not depend on the second variable, we may regard $u_{0}(x, y)=u_{0}(x)$ and $u_{0} \in H^{1}(0,1)$, concluding the proof.

5.3. Continuity of the equilibria set. In this section, we show our main result. First, let us collect some lemmas and propositions in order to achieve our goal.

Lemma 5.3. Let $u^{\varepsilon} \in H^{1}\left(\Omega_{\varepsilon}\right)$ and denote by $w^{\varepsilon} \in H^{1}\left(\Omega_{\varepsilon}\right)$ the function $w^{\varepsilon}=A_{\varepsilon}^{-1} F_{\varepsilon}\left(u^{\varepsilon}\right)$. Then $\left\|w^{\varepsilon}\right\|_{H^{1}\left(\Omega_{\varepsilon}\right)} \leq$ $C$ for some $C>0$ independent of $\varepsilon$.

Proof. Since $w^{\varepsilon}=A_{\varepsilon}^{-1} F_{\varepsilon} u^{\varepsilon}$, it follows that, for any $\varphi \in H^{1}\left(\Omega_{\varepsilon}\right)$,

$$
\int_{\Omega_{\varepsilon}} \frac{\partial w^{\varepsilon}}{\partial x_{1}} \frac{\partial \varphi}{\partial x_{1}}+\int_{\Omega_{\varepsilon}} \frac{\partial w^{\varepsilon}}{\partial x_{2}} \frac{\partial \varphi}{\partial x_{2}}+\int_{\Omega_{\varepsilon}} w^{\varepsilon} \varphi=\frac{1}{\varepsilon} \int_{\theta_{\varepsilon}} f\left(u^{\varepsilon}\right) \varphi
$$

Therefore, if we take $\varphi=w^{\varepsilon}$, it follows from Lemma 3.7 that

$$
\left\|w^{\varepsilon}\right\|_{H^{1}\left(\Omega_{\varepsilon}\right)} \leq\left(\frac{1}{\varepsilon} \int_{\theta_{\varepsilon}}\left|f\left(u^{\varepsilon}\right)\right|^{2}\right)^{1 / 2}\left(\frac{1}{\varepsilon} \int_{\theta_{\varepsilon}}\left|w^{\varepsilon}\right|^{2}\right)^{1 / 2} \leq\|f\|_{\infty} h_{1}^{1 / 2}\left\|w^{\varepsilon}\right\|_{H^{1}\left(\Omega_{\varepsilon}\right)} .
$$

Thus, $\left\|w^{\varepsilon}\right\|_{H^{1}\left(\Omega_{\varepsilon}\right)} \leq C$.

Now, we will analyze the asymptotic behavior of the nonlinearities. 
Proposition 5.4. If we have $u^{\varepsilon} \in H^{1}\left(\Omega_{\varepsilon}\right)$ and $u_{0} \in H^{1}(\Omega)$ with $P_{\varepsilon} u^{\varepsilon} \rightarrow u_{0}$ in $H^{1}(\Omega)$ and $u_{0}\left(x_{1}, x_{2}\right)=$ $u_{0}\left(x_{1}\right)$, for all $\left(x_{1}, x_{2}\right) \in \Omega$, then, for all $\varphi \in H^{1}(0,1)$, we have

$$
\frac{1}{\varepsilon} \int_{\theta_{\varepsilon}} f\left(u^{\varepsilon}\right) \varphi \rightarrow \mu_{h} \int_{0}^{1} f\left(u_{0}\right) \varphi
$$

where $\mu_{h}$ is the average of the function $h$ as in Remark 2.1.

Proof. Indeed,

$$
\frac{1}{\varepsilon} \int_{\theta_{\varepsilon}} f\left(u^{\varepsilon}\right) \varphi-\mu_{h} \int_{0}^{1} f\left(u_{0}\right) \varphi=\frac{1}{\varepsilon} \int_{\theta_{\varepsilon}}\left(f\left(u^{\varepsilon}\right)-f\left(u_{0}\right)\right) \varphi+\frac{1}{\varepsilon} \int_{\theta_{\varepsilon}} f\left(u_{0}\right) \varphi-\int_{0}^{1} \mu_{h} f\left(u_{0}\right) \varphi .
$$

Using the definition of $\mu_{h}$ and with standard computations, we have

$$
\begin{aligned}
& \left|\frac{1}{\varepsilon} \int_{\theta_{\varepsilon}} f\left(u^{\varepsilon}\right) \varphi-\mu_{h} \int_{0}^{1} f\left(u_{0}\right) \varphi\right| \leq \frac{1}{\varepsilon} \int_{\theta_{\varepsilon}}\left|f\left(u^{\varepsilon}\left(x_{1}, x_{2}\right)\right)-f\left(u_{0}\left(x_{1}\right)\right)\right|\left|\varphi\left(x_{1}\right)\right| d x_{2} d x_{1} \\
& \quad+\left|\frac{1}{\varepsilon} \int_{0}^{1} \int_{g_{\varepsilon}\left(x_{1}\right)-\varepsilon h_{\varepsilon}\left(x_{1}\right)}^{g_{\varepsilon}\left(x_{1}\right)} f\left(u_{0}\left(x_{1}\right)\right) \varphi\left(x_{1}\right) d x_{2} d x_{1}-\int_{0}^{1} f\left(u_{0}\left(x_{1}\right)\right) \mu_{h} \varphi\left(x_{1}\right) d x_{1}\right| \\
& \leq\left(\frac{1}{\varepsilon} \int_{\theta_{\varepsilon}}\left|f\left(u^{\varepsilon}\left(x_{1}, x_{2}\right)\right)-f\left(u_{0}\left(x_{1}\right)\right)\right|^{2} d x_{2} d x_{1}\right)^{1 / 2}\left(\frac{1}{\varepsilon} \int_{\theta_{\varepsilon}}\left|\varphi\left(x_{1}\right)\right|^{2} d x_{2} d x_{1}\right)^{1 / 2} \\
& \quad+\left|\int_{0}^{1} \frac{1}{\varepsilon}\left[g_{\varepsilon}\left(x_{1}\right)-\left(g_{\varepsilon}\left(x_{1}\right)-\varepsilon h_{\varepsilon}\left(x_{1}\right)\right)\right] f\left(u_{0}\left(x_{1}\right)\right) \varphi\left(x_{1}\right) d x_{1}-\int_{0}^{1} f\left(u_{0}\left(x_{1}\right)\right) \mu_{h} \varphi\left(x_{1}\right) d x_{1}\right|
\end{aligned}
$$

Now, since $\varphi=\varphi\left(x_{1}\right)$ we have $\left.\left|\frac{1}{\varepsilon} \int_{\theta_{\varepsilon}}\right| \varphi\left(x_{1}\right)\right|^{2} d x_{2} d x_{1} \mid \leq h_{1}\|\varphi\|_{L^{2}(0,1)}^{2} \leq C\|\varphi\|_{H^{1}(0,1)}^{2}$. Moreover, using Theorem 3.7. Remark 2.1 and the uniform bound of $f$ and $f^{\prime}$, it follows that, for $1 / 2<s<1$,

$$
\begin{aligned}
& \leq\left\|f^{\prime}\right\|_{\infty} C_{1}\left(\frac{1}{\varepsilon} \int_{\theta_{\varepsilon}}\left|u^{\varepsilon}\left(x_{1}, x_{2}\right)-u_{0}\left(x_{1}\right)\right|^{2} d x_{2} d x_{1}\right)^{1 / 2}\|\varphi\|_{H^{1}(0,1)}+\left|\int_{0}^{1} f\left(u_{0}\left(x_{1}\right)\right) \varphi\left(x_{1}\right)\left(h_{\varepsilon}\left(x_{1}\right)-\mu_{h}\right) d x_{1}\right| \\
& \leq K\left\|u^{\varepsilon}-u_{0}\right\|_{X_{\varepsilon}}+\left|\int_{0}^{1} f\left(u_{0}\left(x_{1}\right)\right) \varphi\left(x_{1}\right)\left(h_{\varepsilon}\left(x_{1}\right)-\mu_{h}\right) d x_{1}\right| \\
& \leq \tilde{K}\left\|P_{\varepsilon} u^{\varepsilon}-u_{0}\right\|_{X}+\left|\int_{0}^{1} f\left(u_{0}\left(x_{1}\right)\right) \varphi\left(x_{1}\right)\left(h_{\varepsilon}\left(x_{1}\right)-\mu_{h}\right) d x_{1}\right| \longrightarrow 0 \text { as } \varepsilon \rightarrow 0 .
\end{aligned}
$$

This completes the proof.

We also need a notion of compactness for sequences, and convergence for operators which are defined in different spaces. We follow the exposition from 43 . See also 42.

In general, consider a family of Hilbert spaces $X_{\varepsilon}$ and a limit Hilbert space $X_{0}$. Besides, let $E_{\varepsilon}: X_{0} \rightarrow X_{\varepsilon}$ a family of operators such that $\left\|E_{\varepsilon} u\right\|_{X_{\varepsilon}} \rightarrow\|u\|_{X_{0}}$ when $\varepsilon \rightarrow 0$. We recall that a sequence $u^{\varepsilon} \in X_{\varepsilon}$ $E$-converges to $u_{0} \in X_{0}$, if $\left\|u^{\varepsilon}-E_{\varepsilon} u\right\|_{X_{\varepsilon}} \rightarrow 0$. This will be denoted by $u_{\varepsilon} \stackrel{E}{\rightarrow} u$.

Definition 5.5. A sequence $\left\{u_{n}\right\}, u_{n} \in X_{\varepsilon_{n}}$ with $\varepsilon_{n} \rightarrow 0$, is E-precompact if for all subsequence $\left\{u_{n^{\prime}}\right\}$ there are a subsequence $\left\{u_{n^{\prime \prime}}\right\}$ and an element $u \in X_{0}$ such that $u_{n^{\prime \prime}} \stackrel{E}{\rightarrow} u$. A family is said to be E-precompact is all sequence $\left\{u_{n}\right\}, u_{n} \in X_{\varepsilon_{n}}$ with $\varepsilon_{n} \rightarrow 0$, is E-precompact.

Definition 5.6. We say that a family of operators $\left\{T_{\varepsilon}\right\}$, with $T_{\varepsilon}: X_{\varepsilon} \rightarrow X_{\varepsilon}$, E-converges to $T: X_{0} \rightarrow X_{0}$ when $\varepsilon \rightarrow 0$ if $T_{\varepsilon} u^{\varepsilon} \stackrel{E}{\rightarrow} T u$ for any $u^{\varepsilon} \stackrel{E}{\rightarrow} u$. We denote this convergence by $T_{\varepsilon} \stackrel{E E}{\longrightarrow} T$.

Finally, we may define a notion of compact convergence for operators.

Definition 5.7. A family of compact operators $\left\{T_{\varepsilon}\right\}$, with $T_{\varepsilon}: X_{\varepsilon} \rightarrow X_{\varepsilon}$, converges compactly to $T$ : $X_{0} \rightarrow X_{0}$ when $\varepsilon \rightarrow 0$ if, for any family $\left\{u^{\varepsilon}\right\}$ with $\left\|u^{\varepsilon}\right\|_{H^{1}\left(\Omega_{\varepsilon}\right)}$ uniformly bounded, we have that $\left\{T_{\varepsilon} u^{\varepsilon}\right\}$ is E-precompact and $T_{\varepsilon} \stackrel{E E}{\longrightarrow} T$. We denote this compact convergence by $T_{\varepsilon} \stackrel{C C}{\longrightarrow} T$. 
For now on, consider again the spaces $X_{\varepsilon}$ defined in (4.1). The next result show the compact convergence of the operators $A_{\varepsilon}^{-1}$ to $A_{0}^{-1}$, defined in (5.1), in the Sobolev-Bochner generalized spaces $L^{2}\left(0,1 ; H^{s}\left(0, g_{\varepsilon}\left(x_{1}\right)\right)\right)$.

Proposition 5.8. Using the notation given by (5.1) and 5.2), we have $A_{\varepsilon}^{-1} \stackrel{C C}{\longrightarrow} A_{0}^{-1}$ with $A_{\varepsilon}^{-1}: X_{\varepsilon} \rightarrow X_{\varepsilon}$.

Proof. It will be proved in three parts.

(i) $A_{\varepsilon}^{-1}$ is compact for each $\varepsilon>0$.

Using previous results from, for instance [21, we have $A_{\varepsilon}^{-1}: L^{2}\left(\Omega_{\varepsilon}\right) \rightarrow H^{1}\left(\Omega_{\varepsilon}\right)$ is compact. Hence, since $X_{\varepsilon}$ is continuously embedded in $L^{2}\left(\Omega_{\varepsilon}\right)$, and $H^{1}\left(\Omega_{\varepsilon}\right)$ is compactly embedded in $X_{\varepsilon}$ by Proposition 3.6. we have that

$$
X_{\varepsilon} \stackrel{i}{\longrightarrow} L^{2}\left(\Omega_{\varepsilon}\right) \stackrel{A_{\varepsilon}^{-1}}{\longrightarrow} H^{1}\left(\Omega_{\varepsilon}\right) \stackrel{i}{\longrightarrow} X_{\varepsilon}
$$

Thus, $A_{\varepsilon}^{-1}: X_{\varepsilon} \rightarrow X_{\varepsilon}$ is a family of compact operators for each $\varepsilon>0$. The proof for $\varepsilon=0$ is analogous.

(ii) The family $\left\{A_{\varepsilon}^{-1} f^{\varepsilon}\right\}$ is $E$-precompact when $\left\|f^{\varepsilon}\right\|_{X_{\varepsilon}}$ is bounded.

In fact, if $\left\{f^{\varepsilon}\right\}_{\varepsilon \in(0,1)}$ in $X_{\varepsilon}$ is such that $\left\|f^{\varepsilon}\right\|_{X_{\varepsilon}} \leq M$, define $u^{\varepsilon}:=A_{\varepsilon}^{-1} f^{\varepsilon}$. Then $A_{\varepsilon} u^{\varepsilon}=f^{\varepsilon}$, and $u^{\varepsilon}$ satisfies, for $\varepsilon$ sufficiently small, that

$$
\begin{aligned}
\left\|u^{\varepsilon}\right\|_{H_{\varepsilon}^{1}\left(\Omega_{\varepsilon}\right)}^{2} & \leq \int_{\Omega_{\varepsilon}}\left|f^{\varepsilon} u^{\varepsilon}\right| \leq\left(\int_{\Omega_{\varepsilon}}\left|f^{\varepsilon}\right|^{2}\right)^{1 / 2}\left(\int_{\Omega_{\varepsilon}}\left|u^{\varepsilon}\right|^{2}\right)^{1 / 2} \\
& =\left\|f^{\varepsilon}\right\|_{L^{2}\left(\Omega_{\varepsilon}\right)}\left\|u^{\varepsilon}\right\|_{L^{2}\left(\Omega_{\varepsilon}\right)} \leq\left\|f^{\varepsilon}\right\| X_{\varepsilon}\left\|u^{\varepsilon}\right\|_{H^{1}\left(\Omega_{\varepsilon}\right)} \leq M\left\|u^{\varepsilon}\right\|_{H_{\varepsilon}^{1}\left(\Omega_{\varepsilon}\right)} .
\end{aligned}
$$

Using Proposition 5.2 it follows that $\left\|P_{\varepsilon} u^{\varepsilon}\right\|_{H^{1}(\Omega)}$ is uniformly bounded and there are $u_{0} \in H^{1}(0,1)$ and subsequence, that we will also call $P_{\varepsilon} u^{\varepsilon}$, such that $P_{\varepsilon} u^{\varepsilon} \rightarrow u_{0}$ in $H^{1}(\Omega)$ and, consequently, $P_{\varepsilon} u^{\varepsilon} \rightarrow u_{0}$ in $X$. Furthermore it follows that

$$
\left\|A_{\varepsilon}^{-1} f^{\varepsilon}-E_{\varepsilon} u_{0}\right\|_{X_{\varepsilon}}=\left\|u^{\varepsilon}-E_{\varepsilon} u_{0}\right\|_{X_{\varepsilon}}=\left\|\left.\left(P_{\varepsilon} u^{\varepsilon}-u_{0}\right)\right|_{\Omega_{\varepsilon}}\right\|_{X_{\varepsilon}} \leq\left\|P_{\varepsilon} u^{\varepsilon}-u_{0}\right\|_{X} \rightarrow 0 .
$$

(iii) If $f_{\varepsilon} \stackrel{E}{\rightarrow} f_{0}$, then $A_{\varepsilon}^{-1} f^{\varepsilon} \stackrel{E}{\rightarrow} A_{0}^{-1} f_{0}$.

Indeed, like the previous item, suppose $u^{\varepsilon}:=A_{\varepsilon}^{-1} f^{\varepsilon}$. It follows that $A_{\varepsilon} u^{\varepsilon}=f^{\varepsilon},\left\|f^{\varepsilon}\right\|_{X_{\varepsilon}}$ is bounded, since is $E$-convergent, and then there are again subsequence of $u^{\varepsilon}$ (also called $u^{\varepsilon}$ ) and $u_{0} \in H^{1}(0,1)$ such that $P_{\varepsilon} u^{\varepsilon} \rightarrow u_{0}$ in $H^{1}(\Omega)$.

Since $f_{\varepsilon} \stackrel{E}{\rightarrow} f_{0}$, it follows that

$$
\hat{f}^{\varepsilon}:=\int_{0}^{g_{\varepsilon}\left(x_{1}\right)} f^{\varepsilon}\left(x_{1}, x_{2}\right) d x_{2} \rightarrow f_{0}
$$

in $L^{2}(0,1)$. Thus, using [21, Theorem 4.3] we have that $u_{0} \in H^{1}(0,1)$ satisfies

$$
\int_{0}^{1}\left(-q_{0} u_{0}^{\prime \prime}+u_{0}\right) \varphi=\int_{0}^{1} f_{0} \varphi, \forall \varphi \in H^{1}(0,1)
$$

Furthermore, $A_{0} u_{0}=f_{0}$, that is, $u_{0}=A_{0}^{-1} f_{0}$ and

$$
\left\|A_{\varepsilon}^{-1} f^{\varepsilon}-E_{\varepsilon} A_{0}^{-1} f_{0}\right\|_{X_{\varepsilon}}=\left\|u^{\varepsilon}-E_{\varepsilon} u_{0}\right\|_{X_{\varepsilon}}=\left\|\left.\left(P_{\varepsilon} u^{\varepsilon}-u_{0}\right)\right|_{\Omega_{\varepsilon}}\right\|_{X_{\varepsilon}} \leq\left\|P_{\varepsilon} u^{\varepsilon}-u_{0}\right\|_{X} \rightarrow 0 .
$$

Thus, we conclude the proof.

Now, we prove the result that will guarantee the semicontinuity of the solutions of problem 5.1 .

Proposition 5.9. Using the previous notation, we have $A_{\varepsilon}^{-1} F_{\varepsilon} \stackrel{C C}{\longrightarrow} A_{0}^{-1} F_{0}$ as $\varepsilon \rightarrow 0$.

Proof. We will also divide this proof in three parts.

(a) $A_{\varepsilon}^{-1} F_{\varepsilon}$ is compact for each $\varepsilon>0$, where $A_{\varepsilon}^{-1} F_{\varepsilon}: X_{\varepsilon} \rightarrow X_{\varepsilon}$.

In fact, since $A_{\varepsilon}^{-1}$ is compact by Proposition 5.8 and $F_{\varepsilon}$ is Lipscthiz with constant that is independent of $\varepsilon$ by Proposition $4.1(b)$, the result follows by composing those applications. Thus $A_{\varepsilon}^{-1} F_{\varepsilon}: X_{\varepsilon} \rightarrow X_{\varepsilon}$ is a family of compact operators for each $\varepsilon>0$. The proof for $\varepsilon=0$ is analogous. 
(b) $\left\{A_{\varepsilon}^{-1} F_{\varepsilon}\left(u^{\varepsilon}\right)\right\}$ is $E$-precompact when $\left\|u^{\varepsilon}\right\|_{X_{\varepsilon}}$ is bounded.

Define $z^{\varepsilon}:=A_{\varepsilon}^{-1} F_{\varepsilon}\left(u^{\varepsilon}\right)$ and, consequently, $A_{\varepsilon} z^{\varepsilon}=F_{\varepsilon}\left(u^{\varepsilon}\right)$. Since $u^{\varepsilon} \in X_{\varepsilon}$, we have $z^{\varepsilon} \in H^{1}\left(\Omega_{\varepsilon}\right)$. If we call $f^{\varepsilon}=f\left(u^{\varepsilon}\right) \chi^{\theta_{\varepsilon}} / \varepsilon$ for each $\varepsilon>0$, we obtain $f^{\varepsilon} \in L^{2}\left(\Omega_{\varepsilon}\right)$ and

$$
\int_{\Omega_{\varepsilon}}\left|f^{\varepsilon}\right|^{2}=\frac{1}{\varepsilon} \int_{\theta_{\varepsilon}}\left|f\left(u^{\varepsilon}\right)\right|^{2} \leq\|f\|_{\infty}^{2} h_{1}=K
$$

where $K>0$ is independent of $\varepsilon$. It follows that $\left\|z^{\varepsilon}\right\|_{H_{\varepsilon}^{1}\left(\Omega_{\varepsilon}\right)} \leq K$ and by Proposition 5.2 we have that $\left\|P_{\varepsilon} z^{\varepsilon}\right\|_{H^{1}\left(\Omega_{\varepsilon}\right)}$ is uniformly bounded, where $P_{\varepsilon}$ is the extension operator from Lemma 5.1 .

Also, from Proposition 5.2 , there are $z_{0} \in H^{1}(\Omega)$ and subsequence, that we will also call $P_{\varepsilon} z^{\varepsilon}$, such that $P_{\varepsilon} z^{\varepsilon} \rightarrow z_{0}$ in $X$ and $z_{0}$ is independent of the second variable. Hence, we have $z_{0} \in H^{1}(0,1) \subset X_{0}$.

Thus,

$$
\left\|A_{\varepsilon}^{-1} F_{\varepsilon}\left(u^{\varepsilon}\right)-E_{\varepsilon} z_{0}\right\|_{X_{\varepsilon}}=\left\|z^{\varepsilon}-E_{\varepsilon} z_{0}\right\|_{X_{\varepsilon}}=\left\|\left.\left(P_{\varepsilon} z^{\varepsilon}-z_{0}\right)\right|_{\Omega_{\varepsilon}}\right\|_{X_{\varepsilon}} \leq\left\|P_{\varepsilon} z^{\varepsilon}-z_{0}\right\|_{X} \rightarrow 0 .
$$

(c) $A_{\varepsilon}^{-1} F_{\varepsilon}\left(u^{\varepsilon}\right) \stackrel{E}{\rightarrow} A_{0}^{-1} F_{0}\left(u_{0}\right)$ if $u^{\varepsilon} \stackrel{E}{\rightarrow} u_{0}$.

Arguing as in the previous item, let us define $z^{\varepsilon}:=A_{\varepsilon}^{-1} F_{\varepsilon}\left(u^{\varepsilon}\right)$, and then, $A_{\varepsilon} z^{\varepsilon}=F_{\varepsilon}\left(u^{\varepsilon}\right)$. Since $u^{\varepsilon} \in X_{\varepsilon}$, we have $z^{\varepsilon} \in H^{1}\left(\Omega_{\varepsilon}\right)$. If we call $f^{\varepsilon}=f\left(u^{\varepsilon}\right) \chi^{\theta_{\varepsilon}} / \varepsilon$ for each $\varepsilon>0$, we have

$$
\int_{\Omega_{\varepsilon}}\left|f^{\varepsilon}\right|^{2} d x=\frac{1}{\varepsilon} \int_{\theta_{\varepsilon}}\left|f\left(u^{\varepsilon}\right)\right|^{2} \leq\|f\|_{\infty}^{2} h_{1}=K
$$

with $K>0$ independent of $\varepsilon$. Furthermore, since $u^{\varepsilon} \stackrel{E}{\rightarrow} u_{0}$, if we define

$$
\hat{f} \varepsilon\left(x_{1}\right)=\frac{1}{\varepsilon} \int_{g_{\varepsilon}\left(x_{1}\right)-\varepsilon h_{\varepsilon}\left(x_{1}\right)}^{g_{\varepsilon}\left(x_{1}\right)} f\left(u^{\varepsilon}\left(x_{1}, x_{2}\right)\right) d x_{2},
$$

we have $\hat{f} \varepsilon-\hat{f}$ in $L^{2}(0,1)$ by Proposition 5.4 , with $\hat{f}\left(x_{1}\right)=\mu_{h} f\left(u_{0}\left(x_{1}\right)\right)$. Indeed, for all $\varphi \in L^{2}(0,1)$

$$
\int_{0}^{1}\left(\frac{1}{\varepsilon} \int_{g_{\varepsilon}\left(x_{1}\right)-\varepsilon h_{\varepsilon}\left(x_{1}\right)}^{g_{\varepsilon}\left(x_{1}\right)} f\left(u^{\varepsilon}\right) d x_{2}-\mu_{h} f\left(u_{0}\right)\right) \varphi d x_{1}=\frac{1}{\varepsilon} \int_{\theta_{\varepsilon}} f\left(u^{\varepsilon}\right) \varphi-\int_{0}^{1} \mu_{h} f\left(u_{0}\right) \varphi \rightarrow 0 .
$$

Consequently, from [21, Theorem 4.3] there is $z_{0} \in H^{1}(0,1)$ such that $P_{\varepsilon} z^{\varepsilon} \rightarrow z_{0}$ in $X$, where $z_{0}$ satisfies, for all $\varphi \in H^{1}(0,1)$,

$$
\int_{0}^{1}\left(q_{0} z_{0}^{\prime} \varphi^{\prime}+z_{0} \varphi\right)=\int_{0}^{1} \frac{L_{g}}{\left|Y^{*}\right|} \mu_{g} f\left(u_{0}\right) \varphi
$$

It follows from the definition of $A_{0}$ and $F_{0}$ that $z_{0}=A_{0}^{-1} F_{0}\left(u_{0}\right)$, and then,

$$
\begin{aligned}
\left\|A_{\varepsilon}^{-1} F_{\varepsilon}\left(u^{\varepsilon}\right)-E_{\varepsilon} A_{0}^{-1} F_{0}\left(u_{0}\right)\right\|_{X_{\varepsilon}}=\left\|z^{\varepsilon}-E_{\varepsilon} z_{0}\right\|_{H^{s}\left(\Omega_{\varepsilon}\right)} \\
=\left\|\left.\left(P_{\varepsilon} z^{\varepsilon}-z_{0}\right)\right|_{\Omega_{\varepsilon}}\right\|\left\|_{X_{\varepsilon}} \leq\right\| P_{\varepsilon} z^{\varepsilon}-z_{0} \|_{X} \rightarrow 0
\end{aligned}
$$

concluding the proof.

As a consequence of Proposition 5.9, we can get the following proposition:

Proposition 5.10. For any family of solutions $\left\{u_{*}^{\varepsilon}\right\}$ of (5.1), there is $u_{*}$ solution of (5.2) and a subsequence of $u_{*}^{\varepsilon}$, also called $u_{*}^{\varepsilon}$, such that $u_{*}^{\varepsilon} \stackrel{E}{\rightarrow} u_{*}$.

Proof. It is a direct consequence of [42, Corollary 5.2] or [45, Proposition 5.6].

We also get the reciprocal of the previous proposition when the limit solution is hyperbolic.

Proposition 5.11. If the solution $u^{*}$ of 5.2 is hyperbolic, then there is a sequence $\left\{u_{*}^{\varepsilon}\right\}$ of solutions of (5.1) such that $u_{*}^{\varepsilon} \stackrel{E}{\rightarrow} u^{*}$.

Proof. It follows from [42, Corollary 5.3] or [45, Proposition 5.7]. 
Remark 5.12. In the case when all equilibria points from the limit equation (5.2) are hyperbolic, we have that all of them are isolated, and then, there exists only a finite number of them (see 45., Corollary 5.4 or Proposition 5.5]).

Furthermore, the previous results prove the upper and lower semicontinuity of the equilibrium set at $\varepsilon=0$.

Proof of Theorem 2.4. The item (a) follows from Proposition 5.10, and the item $(b)$ is a consequence of Proposition 5.11.

\section{Final COnClusion}

In this work, we obtain the homogenized equation of a family of solutions of a semilinear elliptic equation, with homogeneous Neumann boundary condition, posed in a two-dimensional oscillating thin region with reaction terms concentrated in a neighborhood of the oscillatory boundary. The main challenge of our analysis is the proof of Theorem 3.7 which gives us estimates for the concentrated integrals. Such result allows us applying, in an appropriated way, mathematical methods and techniques such as Fixed Point Theorem, compact convergence and homogenization theory to pass to the limit in the perturbed equation (2.1) obtaining upper and lower semicontinuity of the set of solutions. The main result of the paper is Theorem 2.4. It is a rigorous convergence result which allows us approximating the solutions of the perturbed equation (2.1) by the non-singular and one-dimensional equation (2.2). Also, we have that the homogenized equation captures the effects of all relevant physical processes that take place in the original problem.

Acknowledgements. The first author (JMA)* is partially supported by grants MTM2016-75465, ICMAT Severo Ochoa project SEV-2015-0554, MINECO, Spain and Grupo de Investigación CADEDIF, UCM. The second author $(\mathrm{AN})^{\diamond}$ was supported by CNPq 141869/2013-5, Brazil. Third one (MCP) $)^{\dagger}$ is partially supported by CNPq 303253/2017-7 and FAPESP 2017/02630-2 Brazil.

\section{REFERENCES}

[1] Scheffer, M. Ecology of Shallow Lakes, Chapman Hall, 1998.

[2] Mocenni, C; Sparacino, E. Identification and simulation of a spatial ecological model in a lake with fractal boundary. Mathematics and Computers in Simulation 79 (2009) 3534-3546.

[3] Chupin, L. The FENE model for viscoelastic thin film flows. Meth. and Appl. of Anal. 16 (2) (2009) 217-262.

[4] Maeda M; Suzuki, K. Concentration of least-energy solutions to a semilinear Neumann problem in thin domains. J. Math. Anal. Appl. 411 (2014) 465-484.

[5] Bressloff, P C. Propagation of CaMKII translocation waves in heterogeneous spiny dendrites. J. Math. Biol. 66 (2013) 1499-1525.

[6] I. Pazanin, I; Suárez-Grau, F J. Effects of rough boundary on the heat transfer in a thin-film flow. Comptes Rendus Mécanique 341 (8) (2013) 646-652.

[7] Boukrouche, M; Ciuperca, I. Asymptotic behaviour of solutions of lubrication problem in a thin domain with a rough boundary and Tresca fluid-solid interface law. Quart. Appl. Math. 64 (2006) 561-591.

[8] Mel'nyk, T A. A mathematical model of the atherosclerosis development in thin blood vessels and its asymptotic approximation. arXiv:1706.00246.

[9] Mocenni, C; Sparacino, E.; Zubelli, J. P. Effective rough boundary parametrization for reaction-diffusion systems. Applicable Analysis and Discrete Mathematics 8 (2014), 33 ?59.

[10] Cioranescu, D and Jean Paulin, J S. Homogenization of Reticulated Structures. Springer-Verlag, 1980.

[11] Tartar, L. The General Theory of Homogenization. A personalized introduction. Lecture Notes of the Unione Matematica Italiana, 7. Springer-Verlag, 2009.

[12] Hale, J H; Raugel, G. Reaction-diffusion equation on thin domains. J. Math. Pures et Appl. (9) 71 (1) (1992) $33-95$.

[13] Raugel, G. Dynamics of partial differential equations on thin domains. Lect. Notes in Math. 1609, Springer-Verlag, 1995.

[14] Elsken, T. Continuity of attractors for net-shaped thin domain. Topol. Meth. Nonlinear Analysis 26 (2005) 315-354.

[15] Prizzi, M; Rybakowski, K P. The effect of domain squeezing upon the dynamics of reaction-diffusion equations. J. of Diff. Equations 173 (2) (2001) 271-320. 
[16] Prizzi, M; Rinaldi, M; Rybakowski, K P. Curved thin domains and parabolic equations, Studia Mathematica 151 (2) (2002) 109-140.

[17] Pereira, M C; Silva, R P. Remarks on the p-Laplacian on thin domains. Progress in Nonlinear Diff. Eq. and Their Appl. (2015) 389-403.

[18] Silva, R P. Global attractors for quasilinear parabolic equations on unbounded thin domains. Monatshefte fur Mathematik 180 (2016) 649-660.

[19] Mel'nyk, T A; Popov, A V. Asymptotic analysis of boundary value and spectral problems in thin perforated domains with rapidly changing thickness and different limiting dimensions. Mat. Sb. 203 (8) (2012) 97-124.

[20] Blanchard, D; Gaudiello, A; Griso, G. Junction of a periodic family of elastic rods with a thin plate. Part II. J. Math. Pures et Appl. 88 (2) (2007) 149-190.

[21] Arrieta, J M; Carvalho, A N; Pereira, M C; Silva, R P. Semilinear parabolic problems in thin domains with a highly oscillatory boundary. Nonlinear Analysis: Theory, Methods \& Applications 74 (2011) 5111-5132.

[22] Arrieta, J M; Pereira, M C. Homogenization in a thin domain with an oscillatory boundary. J. Math. Pures et Appl. 96 (2011) 29-57.

[23] Arrieta, J M; Pereira, M C. The Neumann problem in thin domains with very highly oscillatory boundaries. J. Math. Anal. Appl. 404 (2013) 86-104.

[24] Arrieta, J M; Villanueva-Pesqueira M. Thin domains with doubly oscillatory boundaries. Math. Meth. Appl. Sci. 37 (2) (2014) 158-166.

[25] Pereira, M C; Silva, R P. Correctors for the Neumann problem in thin domains with locally periodic oscillatory structure. Quart. of Appl. Math. 73 (2015) 537-552.

[26] Pereira, M C. Parabolic problems in highly oscillating thin domains. Annali di Matematica Pura ed Applicata 194 (4) (2015) 1203-1244.

[27] Arrieta, J M; Villanueva-Pesqueira M. Unfolding operator method for thin domains with a locally periodic highly oscillatory boundary. SIAM J. of Math. Analysis 48-3 (2016) 1634-1671.

[28] Arrieta, J M; Villanueva-Pesqueira M. Thin domains with non-smooth oscillatory boundaries. J. Math. Anal. Appl. 446 (2017) $130-164$.

[29] A. Gaudiello, K. Hamdache, The polarization in a ferroelectric thin film: local and nonlocal limit problems. ESAIM Control Optim. Calc. Var. 19 (2013) 657-667.

[30] A. Gaudiello, K. Hamdache, A reduced model for the polarization in a ferroelectric thin wire. NoDEA Nonlinear Differential Equations Appl. 22 (6) (2015) 1883-1896.

[31] Pereira, M C; Rossi, J D. Nonlocal problems in thin domains. Journal of Diff. Equations 263 (3) (2017) 1725-1754.

[32] Pereira, M C; Rossi, J D. Nonlocal evolution problems in thin domains. To appear in Applicable Analysis doi.org/10.1080/00036811.2017.1350850.

[33] Arrieta J M; Jiménez-Casas A; Rodríguez-Bernal A. Flux terms and Robin boundary conditions as limit of reactions and potentials concentrating at the boundary. Revista Matemática Iberoamericana. 24 (1) (2008) 183-211.

[34] Jiménez-Casas A; Rodríguez-Bernal A. Asymptotic behaviour of a parabolic problem with terms concentrated in the boundary. Nonlinear Analysis: Theory, Methods \& Applications 71 (2009) 2377-2383.

[35] Jiménez-Casas A; Rodríguez-Bernal A. Singular limit for a nonlinear parabolic equation with terms concentrating on the boundary. J. of Math. Anal. Appl. 379 (2) (2011) 567-588.

[36] Aragão, G S; Bruschi, S M. Limit of nonlinear elliptic equations with concentrated terms and varying domains: the non uniformly Lipschitz case. Electron. J. Differential Equations 217 (2015).

[37] Aragão, G S.; Bruschi, S M. Concentrated terms and varying domains in elliptic equations: Lipschitz case. Math. Methods Appl. Sci. 39 (12) (2016) 3450-3460.

[38] Aragão, G S; Pereira A L; Pereira, M C. A nonlinear elliptic problem with terms concentrating in the boundary. Math. Methods Appl. Sci. 35 (9) (2012) 1110-1116.

[39] Aragão, G S; Pereira A L; Pereira, M C. Attractors for a nonlinear parabolic problem with terms concentrating in the boundary. Journal of Dynamics and Differential Equations, v. 26 (4) (2014) 871-888.

[40] Barros, S R M; Pereira, M C. Semilinear elliptic equations in thin domains with reaction terms concentrating on boundary. J. Math. Anal. Appl., 441(1) (2016) 375-392.

[41] Cioranescu D; Donato P. An introduction to homogenization. Oxford Lecture Series in Mathematics and its Applications vol.17. Oxford University Press, New York, 1999.

[42] Arrieta J M, Bruschi S M. Rapidly varying boundaries in equations with nonlinear boundary conditions. The case of a Lipschitz deformation. Math. Models and Meth. in Appl. Sciences 17 (10) (2007) 1555-1585.

[43] Carvalho, A N; Piskarev, S. A general approximation scheme for attractors of abstract parabolic problems. Numer. Funct. Anal. Optim. 27 (2006) 785-829.

[44] Cazenave, T; Haraux, A. An introduction to semilinear evolution equations. Vol. 13. Oxford University Press on Demand, 1998.

[45] Arrieta, J M, Alexandre N C, Lozada-Cruz, G. Dynamics in dumbbell domains I. Continuity of the set of equilibria. Journal of Differential Equations 231 (2) (2006) 551-597.

[46] Pereira, M C. Asymptotic analysis of a semilinear elliptic equation in highly oscillating thin domains. Zeitschrift fur Angewandte Mathematik und Physik 67 (2016) 1-14. 
[47] Aragão, G S; Pereira, A L; Pereira, M C. Attractors for a nonlinear parabolic problem with terms concentrating on the boundary. J. Dynam. Differential Equations 26 (2014) 871-888.

[48] Grisvard, P. Elliptic problems in nonsmooth domains. Society for Industrial and Applied Mathematics, 2011.

[49] Yagi, A. Abstract parabolic evolution equations and their applications. Springer Science Business Media, 2009.

[50] Meier, S A; Böhm, M. A note on the construction of function spaces for distributed-microstructure models with spatially varying cell geometry. Int. J. Numer. Anal. Model 5.5 (2008) 109-125.

[51] Chandler-Wilde, S N; Hewett, D P; Moiola, A. Interpolation of Hilbert and Sobolev spaces: Quantitative estimates and counterexamples. Mathematika 61.2 (2015) 414-443.

[52] Cazenave, T; Haraux, A. An introduction to semilinear evolution equations. Vol. 13. Oxford Univ. Press on Demand, 1998.

[53] Evans, L C. Partial Differential Equations. Graduate studies in mathematics. American Mathematical Society, 1998.

José M. Arrieta

Dpto. de Análisis Matemático y Matemática Aplicada

Universidad Complutense de Madrid, 28040 Madrid, Spain

AND

Instituto de Ciencias Matemáticas, CSiC-UAM-UC3M-UCM

C/ Nicolás Cabrera 13-15, 28049 Madrid, Spain.

E-mail address: arrieta@mat.ucm.es

Ariadne Nogueira

Dpto. de Matemática, ime, Universidade de São Paulo,

Rua do Matão 1010, São Paulo - SP, Brazil.

E-mail address: ariadnen@ime.usp.br

Marcone C. Pereira

Dpto. de Matemática Aplicada, IMe, Universidade de São Paulo,

Rua do Matão 1010, SÃo Paulo - SP, Brazil.

E-mail address: marcone@ime.usp.br 